\title{
Computational Fluid Dynamics of Catalytic Reactors
}

\author{
Matthias Hettel†, Martin Wörner‡, Olaf Deutschmann†† \\ $†$ Institute for Chemical Technology and Polymer Chemistry \\ \#Institute of Catalysis Research and Technology \\ Karlsruhe Institute of Technology (KIT), Engesserstraße 20, 76128 Karlsruhe, Germany \\ Email: deutschmann@kit.edu
}

\section{Introduction}

Today, the challenge in chemical and material synthesis is not only the development of new catalysts and supports to synthesize a desired product, but also the understanding of the interaction of the catalyst with the surrounding reactive flow field. Often, only the exploitation of these interactions can lead to the desired product selectivity and yield. Hence, a better understanding of gas-solid, liquidsolid and gas-liquid-solid flows in chemical reactors is understood as a critical need in chemical technology calling for the development of reliable simulation tools that integrate detailed models of reaction chemistry and computational fluid dynamics (CFD) modeling of macro-scale flow structures.

The ultimate objective of CFD simulations of catalytic reactors is (1) to understand the interactions of physics (mass and heat transport) and chemistry in the reactor, (2) to support reactor design and engineering, and (3) eventually, to find optimized operating conditions for the maximization of the yield of the desired product and minimization of undesired side-products or pollutants.

This chapter introduces the application of CFD simulations to obtain a better understanding of the interactions between mass and heat transport and chemical reactions in reactors for chemical and materials synthesis, in particular in the presence of catalytic surfaces. Catalytic reactors are generally characterized by the complex interaction of various physical and chemical processes. Bundles or tubular fixed bed reactors can serve as an example. They are widespread in chemical industry and in particular used for reactions with large heat release or supply. Figure 1 illustrates typical time scales and length scales within such a catalytic tube bundle reactor. 

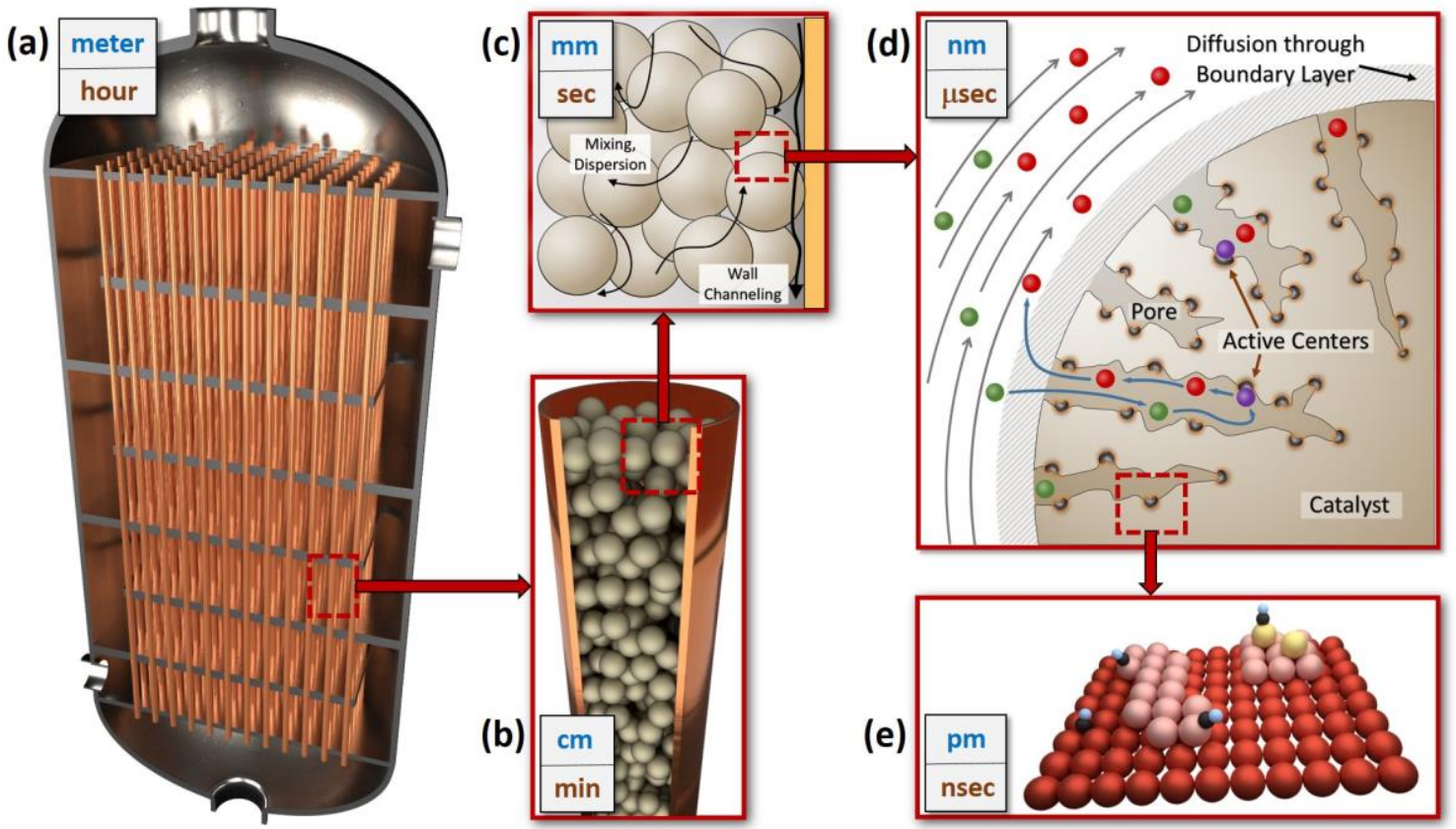

Fig. 1. Typical time scales (blue text) and length scales (brown text) in a catalytic bundled tubes reactor. (a) Principle of the reactor. The mixture flows from top to bottom through a bundle of tubes where the conversion takes place. The tubes are bathed by a fluid (gaseous or liquid) which is guided around the tubes in a crossflow arrangement. This fluid serves as a cooling or heating agent depending on the type of reaction (exothermic or endothermic). The tubes (b) are filled with catalytic particles. In each tube, the transport of momentum, energy, and chemical species occurs not only in flow direction, but also into radial direction (c). Channeling in the near-wall region affects the radial heat transfer; radiation plays a significant role at higher temperatures; local kinetics influence local transport phenomena, and vice versa; reaction rates are limited due to film diffusion. The catalyst material is often dispersed in porous structures like washcoats or pellets (d). Mass transport in the fluid phase and chemical reactions are superimposed by diffusion of the species to the active catalytic centers in the pores. The temperature distribution depends on the interaction of heat convection and conduction in the fluid, heat release due to chemical reactions, heat transport in the solid material, and thermal radiation. The chemical reaction itself takes place at active sites on the inner surface of the porous catalyst structure (e).

Computational fluid dynamics (CFD) is able to predict complex flow fields, even combined with heat transport, due to the recently developed numerical algorithms and the availability of fast computer hardware. The consideration of detailed models for chemical reactions, in particular for heterogeneous reactions, however, is still very challenging due to the large number of species mass conservation equations, their highly non-linear coupling, and the wide range of time scales introduced by the complex reaction networks.

In the following, concepts for modeling and numerical simulation of catalytic reactors are presented, which describe the coupling of the physical and chemical processes in detail. The elementary kinetics and dynamics as well as ways for modeling the intrinsic chemical reactions rates (micro kinetics) by the Mean-Field Approximation (MF) and by lumped kinetics are discussed. It is assumed that models exist that can compute the local heterogeneous but also homogeneous reaction rate as a function of the local conditions such as temperature and species concentration in the gas-phase and of the local and temporal state of the surfaces. These chemical source terms are here coupled with the fluid flow and used to numerically simulate the chemical reactor.

The focus is on the principal ideas and the potential applications of CFD in heterogeneous catalysis; textbooks and specific literature are frequently referenced for more details. Specific examples taken 
from literature and our own work will be used for illustration of the state-of-the-art CFD simulation of chemical reactors with heterogeneously catalyzed reactions.

\section{Modeling of reactive flows}

\subsection{Governing equations of multi-component single phase flows}

As long as a fluid can be treated as a continuum, the most accurate description of the flow field of multi-component mixtures is given by the transient three-dimensional (3D) Navier-Stokes equations coupled with the mass continuity, the energy and species transport equations, which will be summarized in this section. More detailed introductions into fluid dynamics and transport phenomena can be found in a number of textbooks (Bird et al. 2001, Kee et al. 2003, Warnatz et al. 1996, Hayes and Kolaczkowski 1997). Other alternative concepts such as the Lattice-Boltzmann method are not discussed here, because the state of development does not allow a broad application.

Governing equations, which are based on conservation principles, can be derived by consideration of the fluid flow for a differential control volume. The principle of mass conservation leads to the mass continuity equation

$$
\frac{\partial \rho}{\partial t}+\frac{\partial\left(\rho \cdot u_{i}\right)}{\partial x_{i}}=0
$$

with $\rho$ being the mass density, $t$ the time, $x_{i}(i=1,2,3)$ are the Cartesian coordinates, and $u_{i}$ the velocity components. The principle of momentum conservation for Newtonian fluids leads to three scalar equations (Navier-Stokes Equations) for the momentum components $\left(\rho \cdot u_{i}\right)$

$$
\frac{\partial\left(\rho \cdot u_{i}\right)}{\partial t}+\frac{\partial\left(\rho \cdot u_{i} \cdot u_{j}\right)}{\partial x_{j}}+\frac{\partial p}{\partial x_{i}}+\frac{\partial \tau_{i j}}{\partial x_{j}}=\rho \cdot g_{i},
$$

where $p$ is the static pressure, $\tau_{i j}$ is the stress tensor, $g_{i}$ are the components of the gravitational acceleration. The above equation is written for Cartesian coordinates. The stress tensor is given as

$$
\tau_{i j}=-\mu \cdot\left(\frac{\partial u_{i}}{\partial x_{j}}+\frac{\partial u_{j}}{\partial x_{i}}\right)+\left(\frac{2}{3} \mu-\kappa\right) \delta_{i j} \cdot \frac{\partial u_{k}}{\partial x_{k}}
$$

Here, $\kappa$ and $\mu$ are the bulk viscosity and mixture viscosity, respectively, and $\delta_{i j}$ is the Kronecker delta, which is unity for $i=j$, else zero. The bulk viscosity vanishes for low-density mono-atomic gases and is also commonly neglected for dense gases and liquids. The coupled mass continuity and momentum equations have to be solved for the description of the flow field.

In multi-component mixtures, not only the flow field is of interest but also mixing of the chemical species and reactions among them, which can be described by an additional set of partial differential equations. Here, the mass $m_{i}$ of each of the $N_{\mathrm{g}}$ gas-phase species obeys a conservation law that leads to

$$
\frac{\partial\left(\rho \cdot Y_{i}\right)}{\partial t}+\frac{\partial\left(\rho \cdot u_{j} \cdot Y_{i}\right)}{\partial x_{j}}+\frac{\partial\left(j_{i, j}\right)}{\partial x_{j}}=R_{i}^{\mathrm{hom}},
$$

where $Y_{i}$ is the mass fraction of species $i$ in the mixture $\left(Y_{i}=m_{i} / m\right.$, with $m$ as total mass) and $R_{i}^{\text {hom }}$ is the net rate of production due to homogeneous chemical reactions. The components $j_{i, j}$ of the diffusion mass flux caused by concentration and temperature gradients are often modeled by the mixtureaverage formulation: 


$$
j_{i, j}=-\rho \cdot \frac{Y_{i}}{X_{i}} \cdot D_{i}^{\mathrm{M}} \cdot \frac{\partial X_{i}}{\partial x_{j}}-\frac{D_{i}^{\mathrm{T}}}{T} \cdot \frac{\partial T}{\partial x_{j}} .
$$

$D_{i}^{\mathrm{M}}$ is the effective diffusion coefficient of species $i$ in the mixture, $D_{i}^{\mathrm{T}}$ is the thermal diffusion coefficient, which is significant only for light species, and $T$ is the temperature. The molar fraction $X_{i}$ is related to the mass fraction $Y_{i}$ using the species molar masses $M_{i}$ by

$$
X_{i}=\frac{1}{\sum_{j=1}^{N g} Y_{j} / M_{j}} \cdot \frac{Y_{i}}{M_{i}}
$$

Heat transport and heat release due to chemical reactions lead to spatial and temporal temperature distributions in catalytic reactors. The corresponding governing equation for energy conservation is commonly expressed in terms of the specific enthalpy $h$ :

$$
\frac{\partial(\rho \cdot h)}{\partial t}+\frac{\partial\left(\rho \cdot u_{j} \cdot h\right)}{\partial x_{j}}+\frac{\partial j_{q, j}}{\partial x_{j}}=\frac{\partial p}{\partial t}+v_{j} \cdot \frac{\partial p}{\partial x_{j}}-\tau_{j k} \cdot \frac{\partial v_{j}}{\partial x_{k}}+S_{\mathrm{h}},
$$

with $S_{\mathrm{h}}$ being the heat source, for instance due to thermal radiation. In multi-component mixtures, diffusive heat transport is significant due to heat conduction and mass diffusion, hence

$$
j_{q, j}=-\lambda \cdot \frac{\partial T}{\partial x_{j}}+\sum_{i=1}^{N g} h_{i} \cdot j_{i, j}
$$

$\lambda$ is the thermal conductivity of the mixture. The temperature is then related to the enthalpy by the definition of the mixture specific enthalpy

$$
h=\sum_{i=1}^{N g} Y_{i} \cdot h_{i}(T)
$$

with $h_{i}$ being the specific enthalpy of species $i$, which is a monotonic increasing function of temperature. The temperature is then commonly derived from Eq. (9) for known $h$ and $Y_{i}$.

Heat transport in solids such as reactor walls and catalyst materials can also be modeled by an enthalpy equation

$$
\frac{\partial(\rho \cdot h)}{\partial t}-\frac{\partial}{\partial x_{j}}\left(\lambda_{s} \cdot \frac{\partial T}{\partial x_{j}}\right)=S_{\mathrm{h}},
$$

where $h$ is the specific enthalpy and $\lambda_{s}$ the thermal conductivity of the solid material. $S_{\mathrm{h}}$ accounts for heat sources, for instance due to heat release by chemical reactions and electric or radiative heating of the solid.

This system of governing equations is closed by the equation of state to relate the thermodynamic variables density $\rho$, pressure $p$, and temperature $T$. The simplest model of this relation for gaseous flows is the ideal gas equation

$$
p=\frac{\rho \cdot R \cdot T}{\sum_{i=1}^{N_{g}} X_{i} \cdot M_{i}}
$$

with the universal gas constant $R=8.314 \mathrm{~J} \mathrm{~mol}^{-1} \mathrm{~K}^{-1}$.

The transport coefficients of the fluid $\mu, D_{i}{ }^{\mathrm{M}}, D_{i}^{\mathrm{T}}$, and $\lambda$ appearing in Eqs. $(3,5,8)$ depend on temperature and mixture composition. They are derived from the transport coefficients of the individual species and the mixture composition by applying empirical approximations. 
The specific enthalpy $h_{i}$ is a function of temperature and can be expressed in terms of the heat capacity

$$
h_{i}=h_{i}\left(T_{\text {ref }}\right)+\int_{T_{\text {ref }}}^{T} c_{p, i}(T) \mathrm{d} T,
$$

where $h_{i}\left(T_{\text {ref }}\right)$ is the enthalphy at reference conditions $\left(T_{\text {ref }}=298.15 \mathrm{~K}, \mathrm{p}_{0}=1\right.$ bar $)$ and $c_{p, i}$ is the specific heat capacity at constant pressure.

\section{Coupling of the flow field with heterogeneous chemical reactions}

Depending on the spatial resolution of the different catalyst structures, e.g. flat surface, gauzes, pellets, embedded in porous media, the species mass fluxes due to catalytic reactions at these structures are differently coupled with the flow field.

\subsection{Modeling of fluid above non-porous catalytic surfaces}

In the simplest case considered, the catalytic surface consists of a single non-porous homogeneous material, e.g. wires or plates made of platinum. The chemical processes at the surface are then coupled with the surrounding flow field by boundary conditions for the species-continuity equations, Eq. (4), at the gas-solid interface:

$$
\vec{n} \cdot\left(\vec{j}_{i}+\rho \cdot \vec{u}_{\mathrm{Stef}} \cdot Y_{i}\right)=R_{i}^{\mathrm{het}} .
$$

Here, $\vec{n}$ is the outward-pointing unit vector normal to the surface, $\vec{j}_{i}$ is the diffusion mass flux of species $i$ as discussed in Eq. (4), and $R_{i}^{\text {het }}$ is the heterogeneous surface reaction rate, which is given per unit geometric surface area $\left(\mathrm{kg} \mathrm{m}^{-2} \mathrm{~s}^{-1}\right)$. It should be noted, that in this case the catalytic surface (red outline in Fig. 2 left) corresponds to the geometrical surface (green outline in Fig. 2 left) of the fluid-solid interphase of the flow field simulation.

The Stefan velocity $\vec{u}_{\text {Stef }}$ occurs at the surface if there is a net mass flux between the surface and the gas phase:

$$
\vec{n} \cdot \vec{u}_{\mathrm{Stef}}=\frac{1}{\rho} \cdot \sum_{i=1}^{N_{\mathrm{g}}} R_{i}^{\mathrm{het}}
$$

At steady-state conditions, this mass flux vanishes unless mass is deposited on the surface, e.g. chemical vapor deposition, or ablated, e.g. material etching. Equation (13) basically means that for $\vec{u}_{\text {Stef }}=0$ the amount of gas-phase molecules of species $i$, which are consumed/produced at the catalyst by adsorption/desorption, have to diffuse to/from the catalytic wall (Eq. 5).
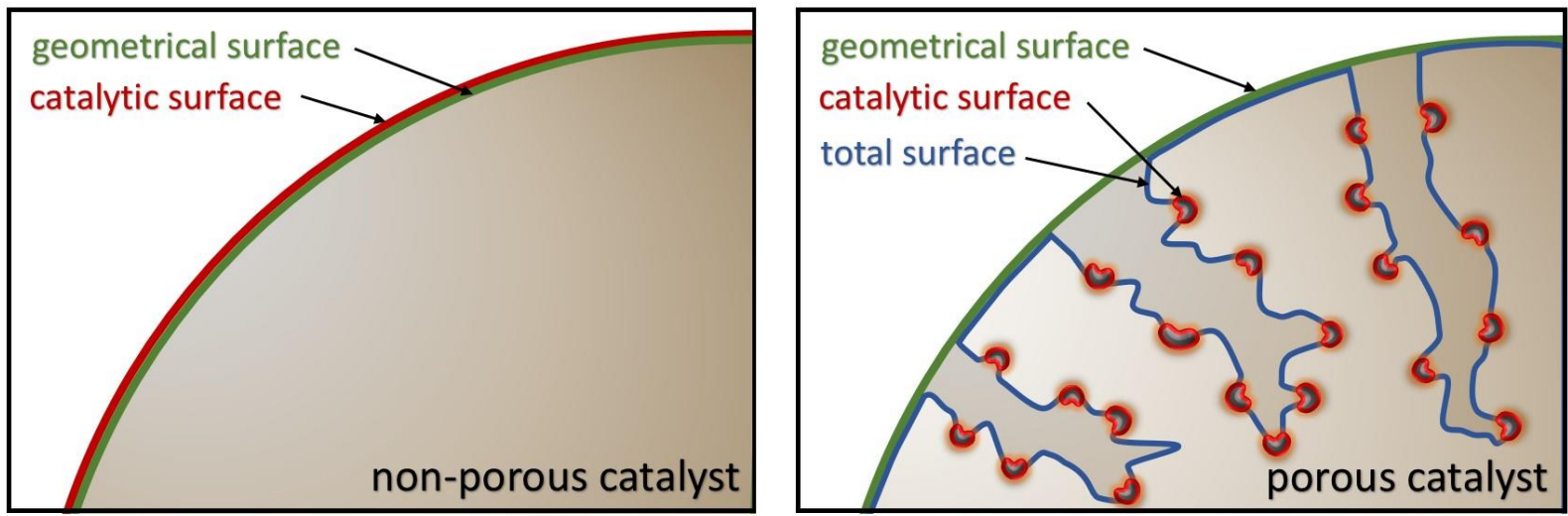

Fig. 2. Left: Definition of surface areas for a non-porous catalyst. Right: Definition of surface areas for porous catalysts (or porous layers such as washcoats). 


\subsection{Fluid flow above porous catalysts}

Most catalysts exhibit a certain structure, for instance, they may occur as dispersed particles on a flat or in a porous substrate. Examples are thin catalytically coated walls in honeycomb structures, foams, disks, plates, and well-defined porous media (e.g. particles). An example is a washcoat (Fig. 3), which is a thin layer of supporting material where small particles of the catalytic active material (e.g. precious metals) is embedded in a supporting material (Fig. 2 right). The numerical grid resolves only the flow region bounded by the geometrical structure of the catalyst. The simplest way to account for the active catalytic surface area consists in scaling the intrinsic reaction of Eq. (14) in the following form:

$$
R_{i}^{\text {het }}=\eta \cdot F_{\text {catgeo }} \cdot M_{i} \cdot \dot{s}_{i} .
$$

Here, $\dot{s}_{i}$ is the molar net production rate of gas phase species $i$, given in mol $\mathrm{m}^{-2} \mathrm{~s}^{-1}$. The area now refers to the actual catalytically active surface area (red outline in Fig. 2 right). The parameter $F_{\text {cat/geo }}$ represents the amount of catalytically active surface area in relation to the geometric surface area of the fluid-solid interphase (green outline in Fig. 2 right). Depending on porosity of the support and the dispersion (e.g. of particles of precious metals) this factor can range up to the order of hundred. The catalytically active surface area is the surface area (layer on which we find adsorbed species) of the catalytic active particles exposed to the ambient gas (fluid) phase. This area can be determined experimentally (e.g. by chemisorption) with sample molecules such as $\mathrm{CO}$ and hydrogen. The catalytically active surface area should not be confused with the BET (Brunauer-Emmett-Teller) surface area representing the total inner surface area of a porous structure (blue outline in Fig. 2 right).

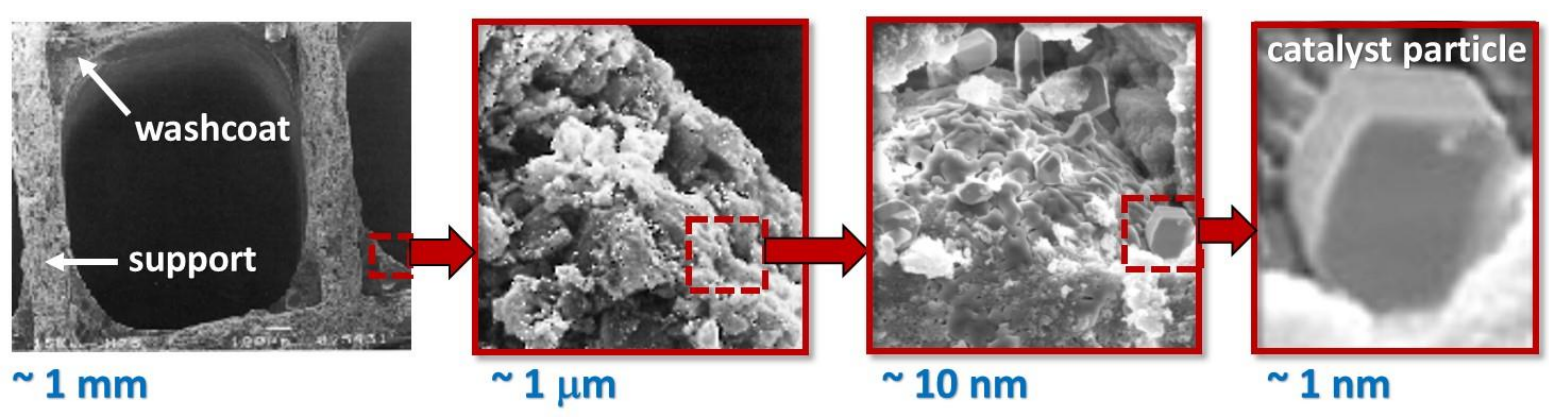

Fig. 3. Washcoat on a channel of a monolith with typical length scales

The simplest model to include the effect of internal mass transfer resistance for catalyst dispersed in a porous media is the effectiveness factor $\eta$ based on the Thiele modulus (Hayes and Kolaczkowski 1997). The effectiveness factor of species $i, \eta_{i}$, is defined as

$$
\eta_{i}=\frac{\dot{s}_{i, \text { mean }}}{\dot{s}_{i}}
$$

with $\dot{s}_{i, \text { mean }}$ as mean surface reaction rate in the porous structure. Assuming a homogeneous porous medium, time-independent concentration profiles, and a rate law of first order, the effectiveness factor can be analytically calculated in terms of

$$
\eta_{i}=\frac{\tanh \left(\Phi_{i}\right)}{\Phi_{i}}
$$

with $\Phi_{i}$ as Thiele module defined as 


$$
\Phi_{i}=L \cdot \sqrt{\frac{\dot{s}_{i} \cdot \gamma}{D_{e f f, i} \cdot c_{i, 0}}} .
$$

Here, $L$ is the thickness of the porous medium (washcoat), $\gamma$ is the ratio of catalytic active surface area to washcoat volume, and $c_{i, 0}$ are the species concentrations at the fluid/porous media interface. The Thiele module is a dimensionless number. The value in the root term of Eq. (18) represents the ratio of intrinsic reaction rate to diffusive mass transport in the porous structure. Since mass conservation has to be obeyed (Eq. 15), the same effectiveness factor has to be applied for all chemical species. Therefore, this simple model can only be applied at conditions, at which the reaction rate of one species determines overall reactivity. Furthermore, this model then implies that mass diffusion inside the porous media can be described by the same diffusion coefficient for all species.

In fixed bed reactors with large numbers of catalytic pellets or for catalyst dispersed in porous media, the structure of the catalyst cannot be resolved geometrically. In those cases, the catalytic reaction rate is expressed per volume, that means $R_{i}^{\text {het }}$ is now given in $\mathrm{kg} \mathrm{m}^{-3} \mathrm{~s}^{-1}$; the volume here refers to the volume of a computational cell in the geometrical domain of a discretized flow region. Then $R_{i}^{\text {het }}$ simply represents an additional source term on the right side of the species-continuity equation, Eq. (4), and is computed by

$$
R_{i}^{\mathrm{het}}=\eta \cdot S_{\mathrm{V}} \cdot M_{i} \cdot \dot{s}_{i}
$$

where $S_{\mathrm{V}}$ is the active catalytic surface area per volumetric unit, given in $\mathrm{m}^{-1}$, determined experimentally or estimated. $F_{\text {cat/geo }}$ as well as $S_{\mathrm{V}}$ can be expressed as a function of the position inside a reactor and time to account for in-homogeneously distributed catalysts and loss of activity, respectively.

\subsection{Porous catalyst as a homogeneous media}

The aforementioned approach fails at conditions under which the reaction rate and diffusion coefficient of more than a single species determines overall reactivity. Hence, the interaction of diffusion and reaction demands more adequate models if mass transport in the porous media is dominated rather by diffusion than by convection. Contrary to the approaches shown above, not only the fluid region has to be discretized, but also the porous solid region that is considered as a homogeneous media.

Concentration gradients inside the porous media result in spatial variations of the surface reaction rates $\dot{s}_{i}$. In thin catalyst layers (washcoats), these are primarily significant in normal direction to the fluid/washcoat boundary. Therefore, one-dimensional reaction-diffusion equations are applied with their spatial coordinate in that direction. Each chemical species leads to one reaction-diffusion equation, which is written in steady state as

$$
\frac{\partial}{\partial r}\left(-D_{i}^{\mathrm{eff}} \cdot \frac{\partial c_{i}^{\mathrm{W}}}{\partial r}\right)-S_{\mathrm{V}} \cdot \dot{s}_{i}=0 .
$$

Here, $c_{i}^{\mathrm{W}}$ denotes the species concentration in the washcoat in normal direction to the boundary fluid/washcoat. $D_{i}^{\text {eff }}$ is the effective diffusion coefficient, which can account for the different diffusion processes in macro and micro pores. 
Eq. (20) is coupled with the surrounding flow field, Eq. (5), at the interface between open fluid and catalytic layer/pellet, where the diffusion fluxes normal to this interface must compensate. In this model the species concentrations, catalytic reaction rates, and surface coverages do not only depend on the position of the catalytic layer/pellet in the reactor, but also vary inside the catalyst layer/particle leading to CPU-time consuming computations.

Fluxes within porous media which are driven by gradients in concentration and pressure, i.e. diffusion and convection, can be described by the Dusty Gas Model (DGM) (Kerkhof and Geboers 2005). This model, which is also applicable for three-dimensional and larger porous media leads to more sophisticated computational efforts.

\subsection{Resolved modeling of porous catalyst}

If a catalytic layer is porous (e.g. washcoat in Fig. 3), the effective catalyst surface area is often significantly larger than the geometrical surface area. The multiplicative factor is $F_{\text {cat/geo }}$ (s. above). Albeit with knowledge of measured properties such as porosity, BET surface area, and chemisorption characteristics, the effective catalyst area is usually adjusted empirically. Moreover, $F_{\text {cat/geo }}$ is often taken to be a constant, independent of changes in reactor operating conditions or age of the catalyst.

Karakaya et al. (2017) performed a detailed analysis of reaction-diffusion processes within a porous rhodium-alumina catalyst washcoat based on a reconstruction of the actual catalyst-support microstructure. In this case, the total surface of a washcoat was used as the catalytic surface area (blue outline in Fig. 2 right). The catalytic chemistry was represented simply as a first-order irreversible reaction $\mathrm{A} \rightarrow \mathrm{B}$, with a rate constant of $k$. The molar net production rate of the gas phase species $\mathrm{B}$ is therefore:

$$
R_{i}^{\text {het }}=F_{\text {geo }} \cdot k
$$

They showed that characteristics as effective area and pore effectiveness are strong functions of the reaction chemistry and the pore microstructure. The results depend greatly on the Damköhler number, which defines the ratio of the of the diffusive time scale to the reaction time scale. At relatively high Damköhler numbers, the reaction is mainly concentrated at the pore openings leaving most of the catalytic area deeper in the pores underutilized. However, as the Damköhler number decreases, the reaction is able to penetrate further into the pores. In this case, the net reaction can depend on local diffusion resistances, such as small necking regions (see Fig. 4-5). In the work of Blasi et al. (2016), the results from the microscale reconstructed pore volumes from the work of Karakaya et al. (2017) were used to develop macroscale models that can be applied at larger length scales. 


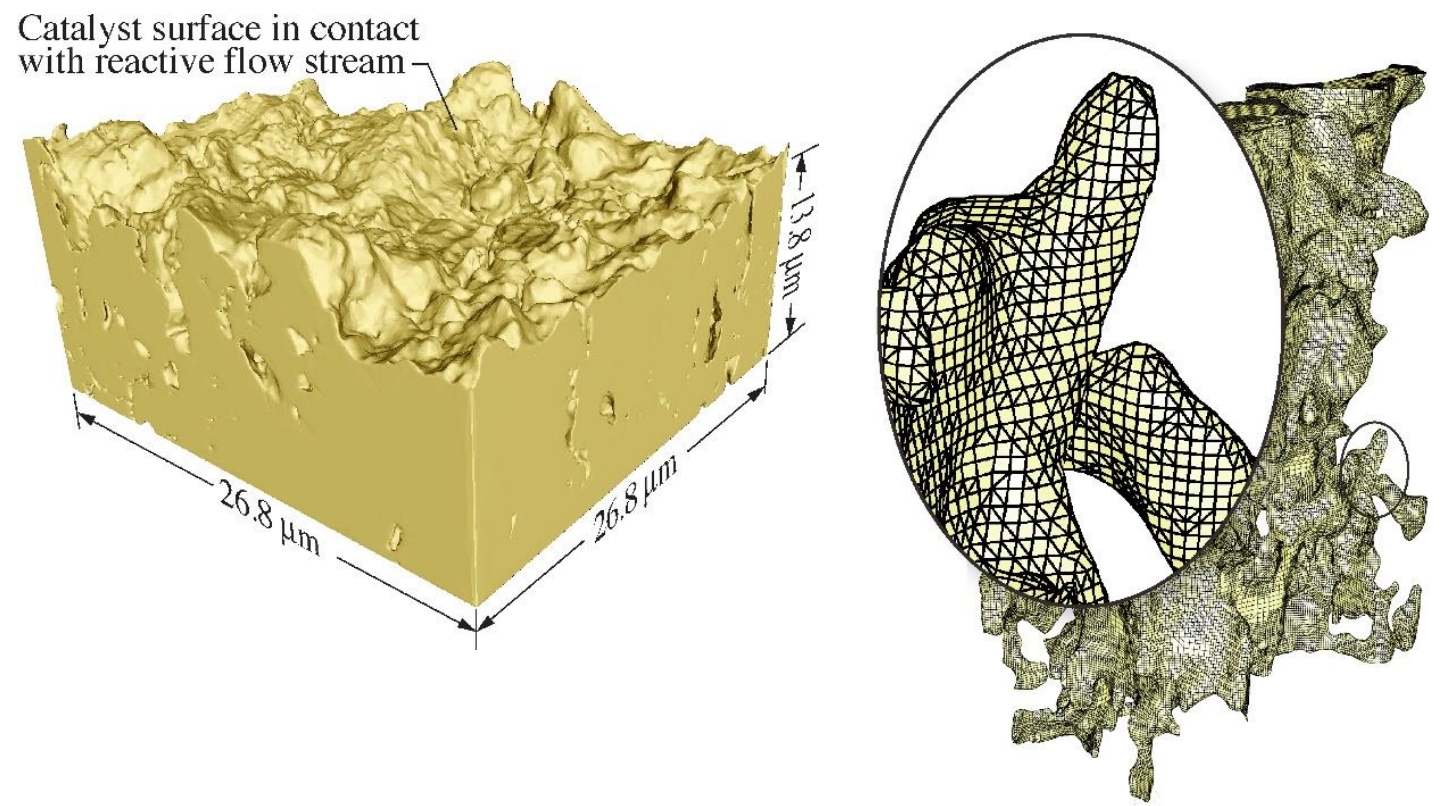

Fig. 4. Left: Three-dimensional reconstruction of a small section of catalyst washcoat. Right: Generated cut-cell mesh of a single pore (Karakaya et al. 2016, reprinted by permission from Elsevier)

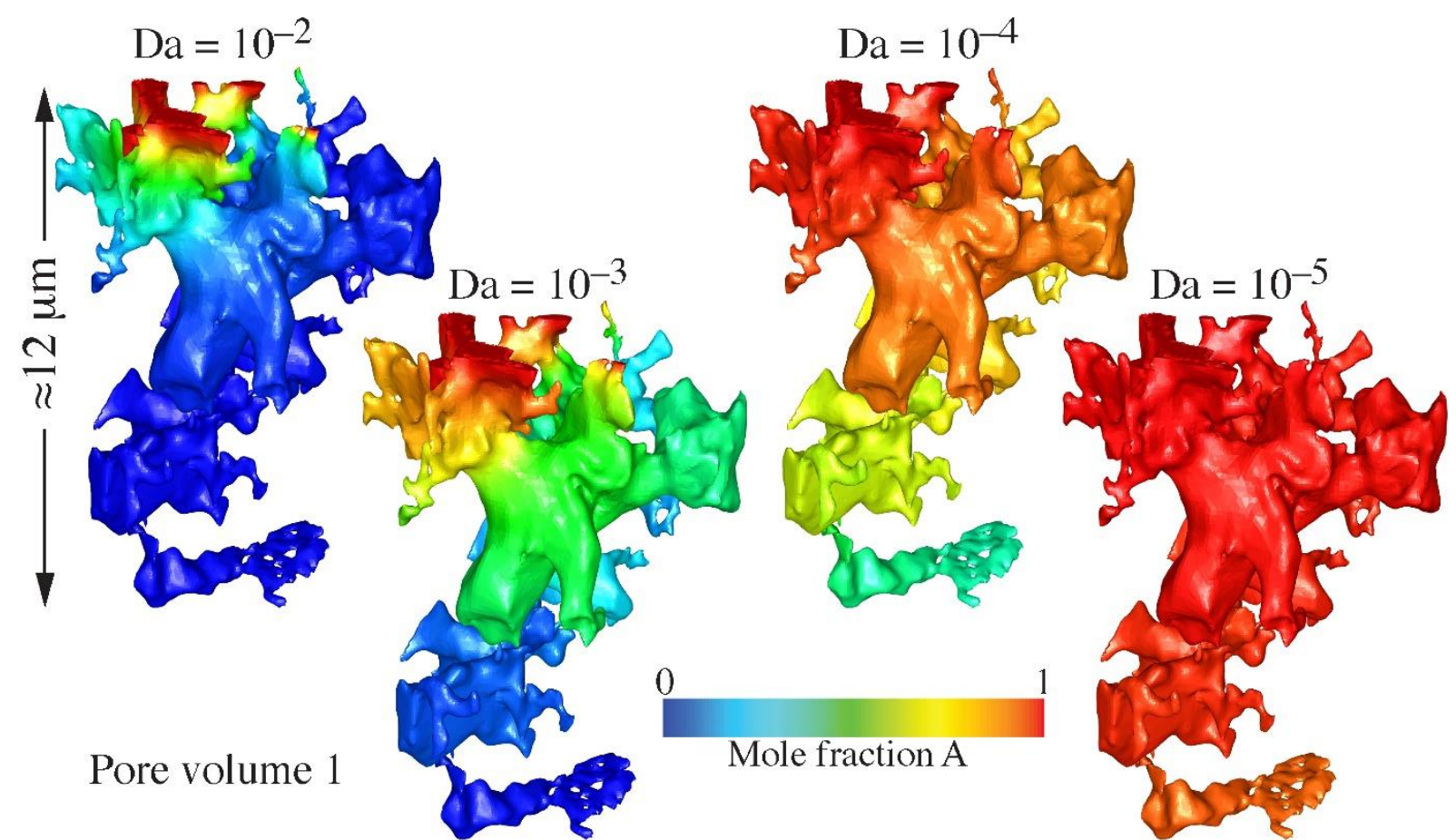

Fig. 5. Predicted reactant mole fractions within a pore at different Damköhler numbers. (Karakaya et al. 2016, reprinted by permission from Elsevier)

\section{Numerical methods and computational tools}

There are a variety of methods to solve the coupled system of partial differential and algebraic equations (PDE), which were presented in the previous sections for modeling catalytic reactors. Very often, the transient three-dimensional governing equations are simplified (no time dependence, symmetry conditions, preferential flow direction, infinite diffusion etc.) as much as possible, but still taking care of all significant processes in the reactor. Simplifications often are not straightforward and need to be conducted with care. Special algorithms were developed for special types of reactors to achieve a converged solution or to speed up the computation solution.

\subsection{Numerical methods for the solution of the governing equations}


An analytic solution of the PDE system is only possible in very limited special cases; for all practical cases, a numerical solution is needed. A numerical solution means, that algebraic equations are derived approximating the solution of the PDE system at discrete points of the geometrical space of the reactor only at discrete points in time. The way of selection of these grid points and the derivation of algebraic equations, which are finally solved by the computer, is called discretization. The grid points are representatives for small flow regions, which are now represented by the cells of a computational grid. Since the solution of the discretized equations is only an approximation of the solution of the PDE system, an error analysis (e.g. a study of grid independency) is an essential feature of the interpretation of every CFD simulation.

\subsection{Turbulence Models}

Turbulent flows are characterized by continuous fluctuations of velocity, which can lead to fluctuations in scalars such as density, temperature, and mixture composition. Turbulence can be desired in catalytic reactors to enhance mixing and reduce mass transfer limitations but is also unwanted due to the increased pressure drop and energy dissipation. An adequate understanding of all facets of turbulent flows is still missing.

Today, the engineer or scientist can choose one of the numerous commercial or public CFD codes including a wide variety of turbulence models. However, the complexity of the codes and the bandwidth of potential applications are enormous and almost nobody is able to overlook all aspects of a modern CFD-code. Even scientists often confine themselves to improve numerical algorithms or to develop models for the description of physical or chemical sub-processes and use the other parts of the codes in good faith of their accurateness. The idea that turbulence or turbulent reaction models may be used without knowledge of the underlying theory is misleading.

This section should give the reader an overview of numerical turbulence and reaction modelling. For a deeper insight some basic additional literature should be mentioned: turbulence (Wilcox 1998; Pope 2000; Lemos 2006), numerical methods (Patankar 1990; Oran and Boris 1987; Fox 1993; Versteeg and Malalasekera 2007), combustion modelling (Libby et al. 1994; Peters 2000; Poinsot and Veynante 2001)

\section{Length and Time scales of turbulent reacting flows}

Turbulent flows are characterized by stochastic fluctuations of velocity, which lead to fluctuations in scalar quantities such as temperature, density and mixture composition. The length scales of turbulent flows range from the size of Kolmogorov eddies $\eta$ up to the size $L_{t}$ (integral length scale) of the large, energy containing eddies. In technical applications, $L_{t}$ is typically one order smaller and $\eta$ typically three orders smaller than the characteristic size of the flow system. Corresponding to the length scales - via a characteristic flow velocity - there exist time scales, accordingly. The time scales of the turbulent flow range from the lifetime of the fine grained Kolmogorov eddies up to the characteristic lifetime $\tau_{t}$ of the large energy containing eddies. On the chemical conversion side, the time scales of reactions range from very small time scales (formation of radicals) up to large time scales (e. g. formation of $\mathrm{NO}_{\mathrm{X}}$ ), and, thus, many time scales exist, that are able to interact with those of turbulent mixture.

\section{Calculation of the flow field}

For the calculation of multidimensional turbulent flows, different methods with various levels of detailedness exist. The most common methods used in CFD are listed in Table 1. The more universal 
a method is, the larger is the computational effort. Besides that, there are numerous specialized methods which are only applicable to distinct flow problems. The acceptable level of complexity is restricted by the available computer capacity.

Tab. 1: Methods for the calculation of fluid dynamics

\begin{tabular}{ll}
\hline Universalism & Method \\
\hline & $\begin{array}{c}\text { Resolution, Solving the Reynolds-averaged Navier-Stokes Equations } \\
\text { Effort }\end{array}$ \\
(RANS-approach) & $\begin{array}{l}\text { 2. Solving the Space-filtered Navier-Stokes Equations } \\
\text { (LES: Large-Eddy-Simulation) } \\
\text { 3. Solving of the Navier-Stokes Equations } \\
\text { (DNS: Direct-Numerical-Simulation) }\end{array}$ \\
\hline
\end{tabular}

Direct numerical simulations (DNS) solve the time-dependent Navier-Stokes-Equations without using any turbulence modelFor technical applications, there are at least three orders of magnitude between the size $L$ of the flow system and the mesh size $\Delta$ needed to resolve the smallest scales. In 3D, this would result in at least $10^{9}$ grid points. . As the length scale of the small vortices decreases with the Reynolds number of the flow, the number of grid cells needed to resolve also the small structures increases. Therefore, DNS is still out of reach as a method to predict even isothermal turbulent flows for practical engineering applications (high Reynolds number) for many years to come. Therefore, this method is restricted to either flows with small Reynolds number (e. g. in the order of some thousands or laminar flows) or to higher Reynolds number but only small calculation domains (in the order of centimeters).

Consequently, turbulent flow configurations are generally modeled with other approaches, i.e., Reynolds-Averaged Navier-Stokes simulations (RANS) or Large-Eddy simulations (LES). Within these two basic approaches, the balance equations are filtered in time or space, respectively. All flow variables are subdivided into a resolved part and an unresolved part. Both approaches introduce additional terms, which have to be modeled to achieve closure, in the Navier-Stokes equations.

The balance equations for RANS (Reynolds-Averaged-Navier-Stokes) techniques are obtained by time averaging the instantaneous balance equations. If the turbulent flow is steady state in the sense that there is no overall time scale of the transient process (statistically steady-state flow, e.g. no periodic variations) the calculation yields only the time averaged quantities. The most popular model is the $k-\varepsilon$ model, which solves two additional transport equations (partial differential equations) for the quantities $k$ (turbulent kinetic energy) and $\varepsilon$ (turbulent dissipation). These two variables are used to determine the turbulent viscosity $\mu_{t} \sim k^{2} / \varepsilon$ representing the effect of the turbulent fluctuations on the exchange of momentum in the flow. This quantity is dependent on the local conditions of turbulence and is no property of the medium. The RANS technique is still the standard approach used for the calculation of catalytic reactors with turbulent flow.

LES is always formulated in a transient way, which makes it a time consuming calculation. In deriving the basic LES equations the instantaneous balance equations are spatially filtered with a filter size of $\Delta$, which usually is the size of the grid. The objective of LES is explicitly to compute the largest structures of the flow field (typically structures larger than the mesh size) whereas the effects that are 
dependent on the motion the smallest eddies are modelled using subgrid closure rules (employing length-scale separation). The evolving local sub grid eddy viscosity $\mu_{t}$ has to be modelled.

Compared to RANS simulations LES simulations are expensive in time and storage space. As modelling is only applied on the smaller unresolved scales the results are usually less sensitive to modelling assumptions as RANS-models. However, usual simplifications, which can be applied by RANS as symmetry conditions or two-dimensional flows cannot be retained. The solution has to be time-dependent, even for statistically steady-state flows.

The Fig. 6 shows schematically the temporal variation of the velocity $u$ at one position in a statistically steady-state flow for different modeling approaches. The DNS claims to predict the same spatial and temporal variations inside the flow as a measurement would yield. The concept of LES implies that the determination of the flow will be the better the finer the grid is. Asymptotically, this leads to a DNS solution. In the frame of a RANS model the velocity is constant in time.

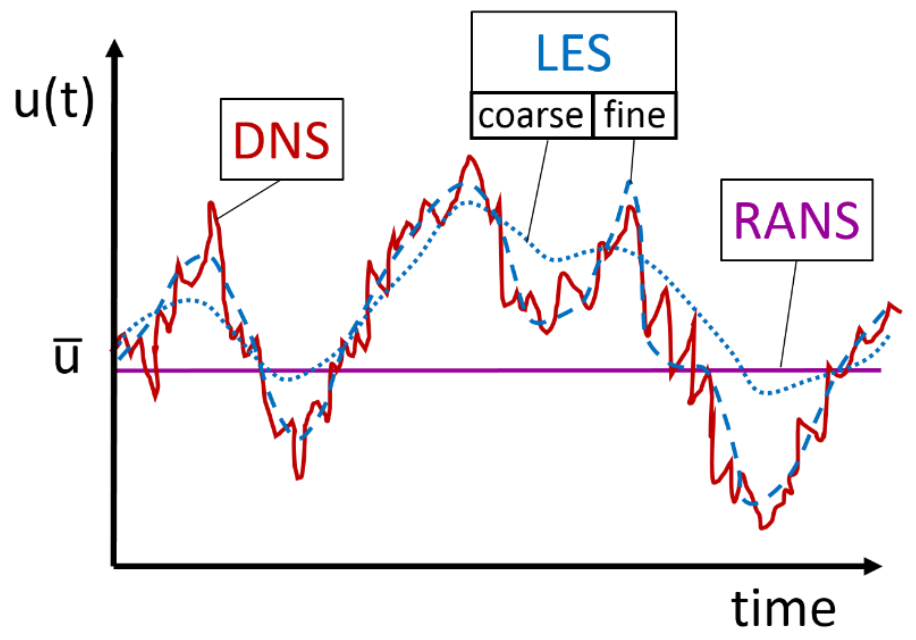

Fig. 6. Velocity $u$ at one position in a statistically steady-state flow for different modeling approaches.

The fluid flow in small-scaled flow regions (in the order of $\mathrm{cm}$ and below) is highly affected by particle surfaces and reactor tube walls, respectively. Consequently, the turbulence intensity is also changed by the presence of a near wall. Getting closer to a wall, the tangential velocity fluctuations are diminished by viscous damping and the normal fluctuations are damped by kinematic blocking. Therefore, it is important to use appropriate models for the flow laminarization at the solid surfaces. The traditional $k-\varepsilon$ model is not suited for near-wall velocity calculation, since the damping in this region is not accounted for. This and some other RANS models are based on wall functions, so that the sublayer and buffer layer do not have to be resolved. This is often enough if the flow region is large scaled. As a consequence, turbulence models that are based on the resolution of the near wall zones have to be used for small scaled flow regions. These are termed low-Reynolds number models as the Spalart-Allmaras and $k-\omega$ model, where $\omega$ is a typical frequency of turbulence. Sometimes the flow region consists of distinct laminar and turbulent zones. An example is an automotive converter, where the flow inside the channels of the monolith is laminar whereas the flow upstream and downstream of the monolith is turbulent. RANS models cannot predict the laminarization in front of and the transition to turbulence behind the monolith.

Contrary, the LES approach is able to resolve the near wall turbulence as well as laminarization and transition processes. Dixon et al. (2011) investigated different RANS turbulence models on a single 
sphere in terms of drag coefficient and heat transfer Nusselt number. As a reference case, Large-Eddy simulations (LES) were carried out showing good agreement with correlations from literature. However, the small time step and fine mesh required are unpractical for entire bed simulations. The authors suggest using the shear-stress transport (SST) $k-\omega$ turbulence model, which is capable to predict heat transfer accurately. Cornejo et al. (2018) calculated the gas flow inside an entire automotive converter applying the porous body approach and using a RANS model. The decay of the turbulence inside of the single channels of the monolith was investigated using a LES model.

\section{Turbulence and Chemistry}

Turbulence implies a large range of both length scales and time scales. This is valid for both flow and chemical reactions. A basic concept for the development of turbulent reaction models is the physical principle stating that the probability of the interaction of scales decreases with the amount of scale separation. There are many circumstances where only limited ranges of chemical and turbulent time scales are involved and where the overlap of these ranges is small. According to Damköhler, the interaction of time scales can be expressed by the ratio of the typical times scales of chemical conversion $\tau_{c}$ and turbulence $\tau_{t}$ called the Damköhler number: $D a=\tau_{c} / \tau_{t}$.

Classical turbulent reaction models describing homogeneous reactions (e.g. combustion) explicitly or implicitly assume scale separation (fast chemistry). If the turbulent mixture is relatively fast or the chemistry is relatively slow (Damköhler number < 1) there is no time-scale separation. In this case PDF-approaches are claimed. Here, probability density functions (PDFs) (Warnatz et al. 1996), either derived by transport equations or empirically constructed, are used to consider the turbulent fluctuations when calculating the chemical source terms. In combination with RANS models for turbulence, these combustion models yield the time-averaged chemical reaction rates (Warnatz et al. 1996; Libby and Williams 1994). The realization of homogeneous reaction in the LES approach is handled in a similar way for the subgrid scales. As the reaction is determined by molecular mixing, reaction takes place only on this dimension of scales.

There is a lack in literature with respect to the modeling of systems where turbulence and heterogeneous chemistry concur. To the best knowledge of the authors, no paper can be found where the impact of turbulence on the chemical conversion on a surface is discussed with respect to the interaction of timescales.

\subsection{CFD software}

The three major approaches of discretization are the Finite Difference Method (FDM), the Finite Volume Method (FVM), and the Finite Element method (FEM). Today, nearly all commercial CFD codes are based on the FVM, only few use the FEM. Currently available multi-purpose commercial CFD codes can simulate very complex flow configurations including turbulence and multi component transport. However, CFD codes still have difficulties to implement complex models for the chemical processes. One problem is the insufficient number of reactions and species the codes can handle. An area of recent development is the implementation of detailed models for heterogeneous reactions.

Several software packages have been developed for modeling complex reaction kinetics in CFD such as CHEMKIN, CANTERA, DETCHEM, which also offer CFD codes for special reactor configurations such as channel flows and monolithic reactors. These kinetic packages and a variety of user written subroutines for modeling complex reaction kinetics have meanwhile been coupled to 
several commercial CFD codes. Aside from the commercially widespread multi-purpose CFD software packages such as FLUENT, STAR-CD, FIRE, CFD-ACE+, CFX, a variety of multi-purpose and specialized CFD codes have been developed in academia and at research facilities. A widespread CFD-code is the tool OpenFOAM (OpenFOAM 2017). By being open-source, OpenFOAM offers users complete freedom to customize and extend its existing functionality.

\section{Reactor simulations}

From the modelling point of view catalytic reactors can be classified by the geometrical design of the catalyst material. This is the main characteristic, because the numerical calculation needs a spatial discretization of the fluid region and of the solid material (surface or whole body). Therefore, it is essential how the catalyst is shaped and if it is immobile or not.

On the one hand, the catalyst can consist of one single continuous solid material that is penetrated by regularly distributed straight channels (as in monoliths or honeycomb structures) or by randomly distributed and shaped voids (as in foams). Here, the catalyst material is mostly deposited on the surface of the support material as a washcoat (s. Fig. 3). This is a thin layer of supporting material where small particles of the catalytic active material (e.g. precious metals) is embedded.

On the other hand, the catalyst can consist of single particles with undefined form (e.g. crushed material) or pellets showing a defined geometrical shape (e.g. spheres or cylinders). The single pieces itself can consist of a coated supporting material or can be build-up of the catalyst material itself. In a packed bed, the particles/pellets are immobile. They touch each other in distinct contact points (for spheres) or contact lines/areas (for pellets) only. In a fluidized bed, the particles are moved by the flow and touch only frequently due to collisions.

This section explores the modelling of catalytic reactors. It starts with an approach, where the solid structure is considered as a porous media. This model needs the smallest effort in computer capacities (memory and time) and can be applied for all of the configurations where the catalyst is immobile. The discretization of both the fluid and the solid structure is more complex and is called resolved modelling in the following. For each of the basic macroscopic morphologies of the catalyst (monolith, foam, fixed bed) some of the most used modeling methods will be presented consecutively. The simulation of mobile catalysts (e.g. fluidized beds) and reactors with multiple fluid phases demands special methods and is discussed afterwards. Various special types of catalysts (e.g. wire gauzes), will not be discussed here.

For all the methods presented for the modeling of a reactor it holds that the microscopic structure of the active part of the catalyst (washcoat or pelleted material) is not resolved by the numerical grid. This is because the morphology of the catalyst layer itself implies very small length scales. The thickness of washcoats is in the order of $0.1 \mathrm{~mm}$ (Fig. 3); the typical size of pores in this material is in the order of $\mathrm{nm}$ and below. However, the modelling of a small part of a reactor (e.g. one channel of a monolith) including the resolved washcoat can lead to very valuable insights in the interaction of processes (see section 3).

\subsection{Modelling of immobile catalyst as a porous media}

A permeable structure can be seen as a multiphase system with one of the phases being a solid matrix. It is considered a continuous media consisting partially of fluid and partially of solid. Within this 
approach, the whole region of the porous body itself is discretized with a numerical grid. That means the real geometry of the flow region or the solid structure is not reproduced by the grid. The volumetric ratio between fluid and solid structure defines the porosity. Using volume averaging leads to a loss of information at the micro-level, but provides insight into the macroscopic effects (e.g. flow maldistribution), which are needed for the full understanding of the system operation.

The flow of fluids through consolidated porous media and through beds of granular solids are similar, both having the general function of pressure drop versus flowrate. Transition from laminar to turbulent flow is gradual. Depending on the Reynolds number based on length scales of the macroscopic pores (e. g. diameter of channels in a monoliths/foams or particle diameter of a fixed bed) four distinct flow regimes can be observed (Dybbs and Edwards 1984): Darcy or creeping flow, steady laminar inertial flow, unsteady laminar flow, highly unsteady and chaotic flow regime. If the Reynolds number is very small, the empirical Darcy's law is applied traditionally for the determination of the pressure loss of the flow. For larger Reynolds number, the models of Ergun, Brinkman, Forchheimer followed by numerous extended models were developed (Lemos 2006). The pressure loss may be strongly anisotropic, depending on the structure of the solid (e.g. for monoliths).

The properties of the porous medium (e.g. heat conductivity) which is a combination of the fluid and the solid part have to be deduced with additional models. Heat transport in porous media involves conductive heat transfer both in solid struts and in the fluid phase as well as convective heat transport due to the flow. The overall conductive heat transport depends on the material and structural properties of the solid and on the properties of the working fluid. Under the assumption of a local thermal equilibrium between fluid and solid phases, mixture models were used traditionally for heat conduction in porous media. By this, the temperatures of solids and fluids are assumed to be the same locally and the heat conduction equations for the solid and fluid phase are lumped together. If the solid and fluid are in thermally non-equilibrium state, the heat conductions in the fluid and solid phases have to be considered with two separate bilance equations. For heat exchange between fluid and solid structure Nusselt number correlations are needed. If the temperature is high, the radiative heat transport has to be included.

The prediction of the mass transfer of species between flow and surface in the case of catalytic reactions demands additional models (Sherwood number correlations or effectiveness factor approaches). Within the porous media approach, the catalytic surface is parametrized as a specific area (square meter surface per volume porous body).

The modelling of a monolith as a continuous porous medium can be found in (Hayes et al. 2012; Porter et al. 2016). By pre-computing the required data at the micro- and mesoscales and storing them in a look-up table, small-scale effects (such as washcoat diffusion) can be captured in a macro-scale model that executes with practicable execution times (Nien et al. 2013). A multiscale model for a fixed bed catalytic reactor is given in (Park 2018). The macroscale part is for the transport across the reactor and the microscale part is for the intra-particle transport and reaction. The effects of pellet size, intra-pellet diffusivity and conductivity on the reactor yield and catalyst effectiveness factor are investigated.

\subsection{Resolved modelling of monoliths (honeycomb-structured catalysts)}

In environmental catalysis (e.g. automotive catalytic converters, high-temperature catalysis, catalytic combustion and reforming) honeycomb-structured catalysts are often applied. Reactors that are based 
on monolithic structures include many equally sized small channels. To resolve these structures with CFD methods in detail, grids with high spatial resolution are needed, which leads to large numbers of grid cells and very large simulation times. To shorten the computer resources needed for modeling of structured catalytic reactors several strategies with various levels of complexity and accuracy have been developed.

One possibility is to model only one representative channel of the whole monolith in 1D/2D/3D with or without inclusion of diffusion effects in the catalytic coating (washcoat). This approach is successful if only one channel is a good representative for all other channels of the monolith. However, a typical monolith consists of thousands of channels running in parallel and the operations conditions for each of them might vary significantly. If the conditions inside the monolith are inhomogeneous it is far better to calculate a certain number of channels and assuming that each of those channels is a representative for a group of them (Tischer and Deutschmann 2005; Sharma and Birgersson 2016; Hettel et al. 2015). Another approach is to exclude the channels of the catalyst from the CFD-domain and to solve them using 1D/2D grids using a second solver (Hettel et al. 2015; Sui et al. 2016).

The works where all channels of a real converter are resolved by the numerical grid include restrictions in dimensionality, complexity of models or in the number of channels considered. Either the number of channels resolved is large (some thousands) and there is no chemistry involved or the number of channels is small (some hundreds) and global chemistry or reduction in dimensionality is used.

Publications presenting the calculation of multi-channel systems resolving the $3 \mathrm{D}$ geometry and using detailed chemistry are scarce in literature. Some papers focus on numerical methods and computer resources (Kumar and Mazumder 2010), others using new computer architectures (Choudary and Mazumder 2014). Generally, there is a lack of publications where the results of numerical calculations are compared to spatially resolved experimental data from inside the channels. In the work of (Hettel et al. 2018), the 3D modeling of a quarter of a CPOX (Catalytic Partial Oxidation)-reformer (diameter $20 \mathrm{~mm}$ ) including two monoliths with ca. 300 channels each is discussed (Fig. 7-8). Detailed chemistry was applied and radiation effects between the two solids was considered. The results are compared to detailed measured data.

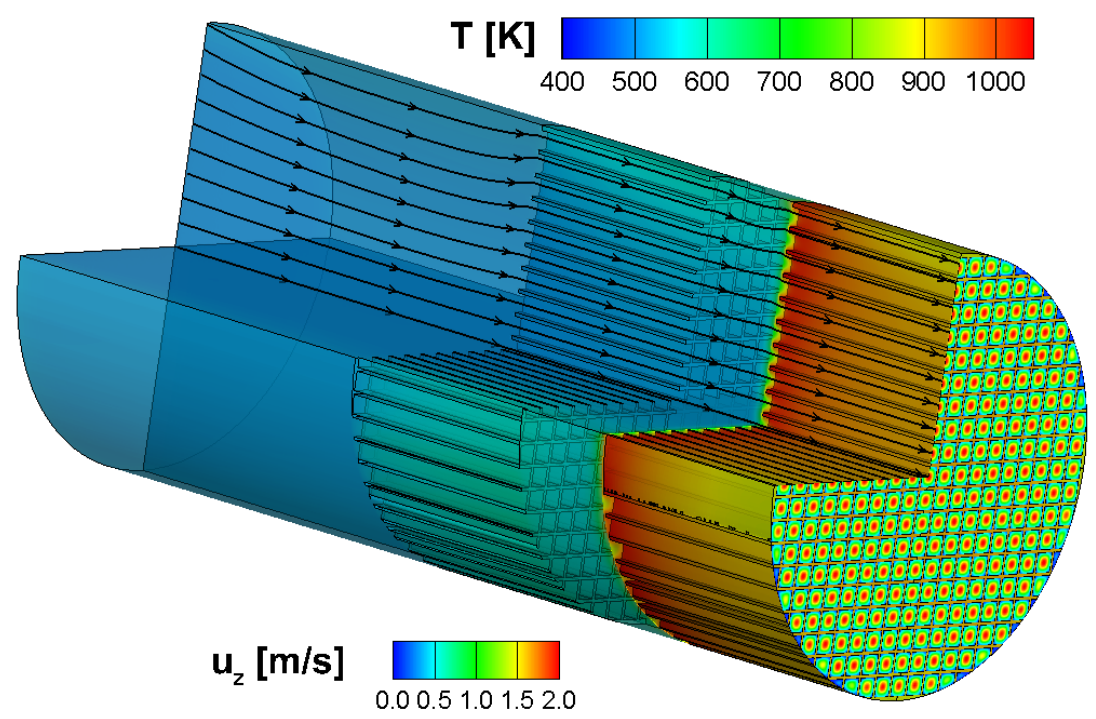


Fig. 7. Temperature field of the fluid part and the two solids (the left monolith is uncoated the right one is coated). Additionally, some streaklines are plotted. Only the slice at the end of the calculation domain shows the distribution of the velocity into z-direction (Hettel et al. 2014, reprinted by permission from Elsevier)

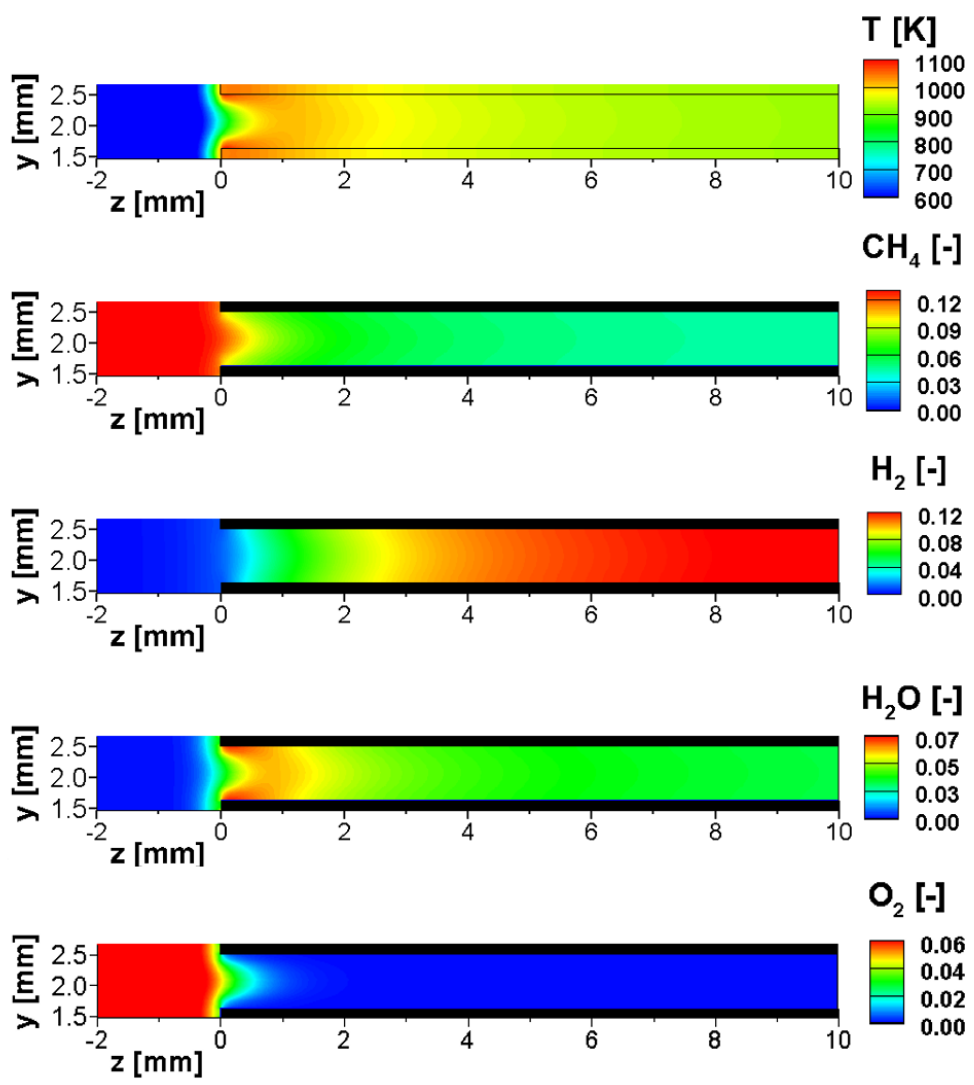

Fig. 8. Intersection through the midplane of only the central channel of the coated monolith. Distributions of temperature and Species (Hettel et al. 2014, reprinted by permission from Elsevier)

\subsection{Resolved modelling of foams}

The application of ceramic foams as catalyst carriers represents a promising alternative to fixed beds or structured packings. Ceramic foams are characterized by their low specific pressure drop, high mechanical stability at relatively low specific weight, enhanced radial transport, as well as a high geometric surface area. Their open-cell structure consists of stiff, interconnected struts building a continuous network. In literature, these structures are typically named as foams or sponges. They can be found in a wide range of technical apparatuses, such as molten metal filters, burner enhancers, soot filters for diesel engine exhausts, and biomedical devices.

Several catalytic applications have been investigated during the last decades (combustion, exhaust gas treatment, syngas production). Maestri et al. (2005) compared by simulation the performance of three different catalyst supports for the CPOX of methane, i.e., honeycomb, foam and fixed-bed reactor. The authors conclude that the foam catalyst support shows the best performance regarding transport properties, syngas selectivity and pressure drop.

The sponge-like structure consists of solid edges and open faces through which fluid can pass. The irregularly shaped strut-network can be expressed by its morphological parameters, i.e., cell and window diameter, strut thickness and porosity. However, industrial manufacturers often use pores per inch (PPI) as a value of characterization, ranging typically between 10-100 and porosity values greater than $75 \%$. One of the most import properties of ceramic foams is the specific surface area, which is significant for the transport of momentum, energy and mass. 
An alternative to transport correlation-based models are structure-resolved 3D CFD simulations. With such detailed simulations, quantitative descriptions can be made of transport phenomena within complex structures without a priori assumptions.

Numerical 3D simulations of foam structures can be divided into real foam models and model structures of different complexity. The first type is carried out by converting the actual geometry by means of 3D Computer Tomography (CT) or 3D magnetic resonance imaging (MRI) into CAD data appropriate for 3D simulations. These models are limited to the scanned structure that is determined by its morphological characteristics. The procedures are time and cost intensive and include several consecutive steps of preparation.

Model structures can be separated into deterministic models (unit cells) and stochastic models. An artificial foam modeler is able to generate structures that can be varied in terms of the most significant morphological characteristics, i.e., porosity, specific surface area and strut dimensions. The modeler does not have to use scanned data but generates the structure automatically, as well as it generates a format that is ready to use for a CFD simulation. However, the usage of idealized unit-cells do not account for random variations typical for natural foam structures. This leads to preferable flow directions in the cell structure. The second type of model structures applies stochastic approaches. For example, a perfectly ordered cell structure can be randomized by relocating nodes with a stochastic algorithm. However, the prediction of pressure drop or residence time is still unsatisfactory. Habisreuther et al. (2009) demonstrated that the parameters porosity and specific surface alone are insufficient to describe the transport phenomena in such complex geometries.

Works showing the modeling of foams including surface reactions are very scarce. Fig. 9 shows examples of the calculation of CPOX in a tubular reactor filled with a foam (see also Wehinger 2016; Wehinger et. al. 2016). 

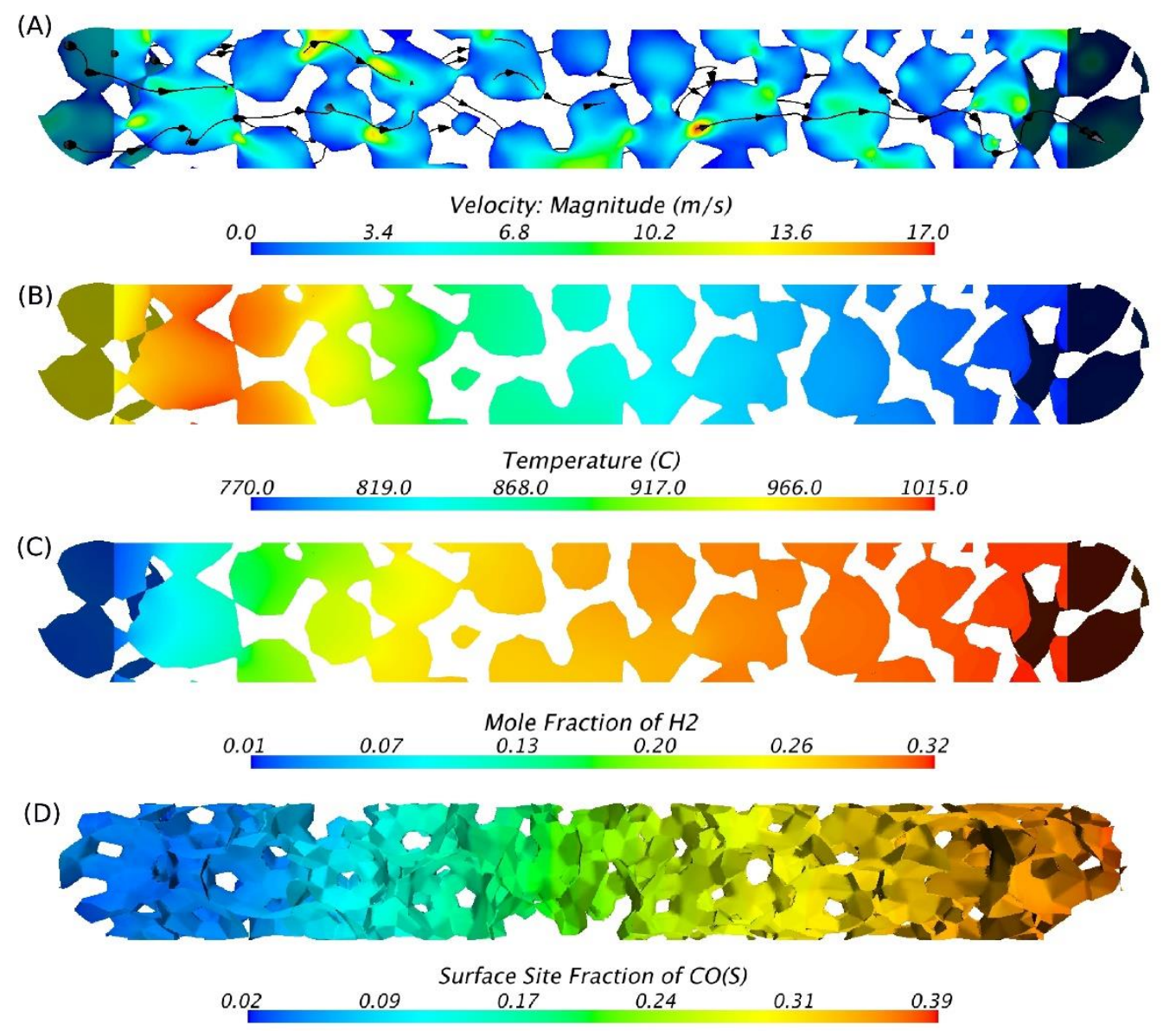

Fig. 9. Modeling of CPOX on a foam coated with Rhodium. (A) velocity magnitude with streamlines, (B) temperature, and (C) hydrogen mole fractions on a plane cut through the CPOX foam. (D) adsorbed $\mathrm{C}^{*}$ on the foam surface (courtesy of G. Wehinger, related papers are: Wehinger 2016; Wehinger et al. 2016)

\subsection{Particle resolved modelling of fixed bed reactors}

A fixed-bed reactor is based on a container filled with randomly positioned particles. The understanding of fluid dynamics and their impact on conversion and selectivity in fixed bed reactors is still very challenging. For large ratios of reactor width to pellet diameter, simple porous media models are usually applicable. This simple approach becomes questionable as this ratio decreases. At small ratios the individual local arrangement of the particles and the corresponding flow field are significant for mass and heat transfer, and, hence, the overall product yields. Therefore, the method of direct numerical simulation (DNS) is the most promising approach. Over the last years, researchers have used particle-resolved CFD simulations to model this kind of packed-bed arrangement more rigorously (Dudukovic 2009). The concept is to take into account the actual shape of each individual particle inside the reactor. Consequently, the fluid flow field is determined by the particles it has to pass by. The advantage is the absence of transport correlations, which are necessary in pseudo-homogeneous or heterogeneous models to describe the transport of momentum, heat and mass. Even though the governing equations are relatively simple for laminar flows, this approach can usually be applied for small and periodic regions of the reactor only, which is caused by the huge number of computational cells needed to resolve all particles.

Several authors used detailed fluid dynamics in combination with pseudo-homogeneous kinetics due to the small number of reaction equations and species (Kuroki et al. 2009). However, this kinetics are often limited to a certain range of process parameters and could therefore not be applied to other flow 
regimes or reactor types. Especially, the species development inside fixed-bed reactors is often insufficiently reproduced with such kinetics in contrast to the exit concentrations (Korup et al. 2011).

For a first-principles approach, modeling spatially resolved fluid dynamics must be combined with reliable kinetics, i.e., microkinetics. Additionally, the diffusion and reaction of the species within the catalyst pellets, which may be made up of micro-porous materials, has to be considered. More recently, a larger bed of catalytic spheres was investigated both experimentally and by particleresolved CFD simulations (Wehinger 2016; Wehinger et. al. 2016b). Fig. 10-11 depict some typical results.
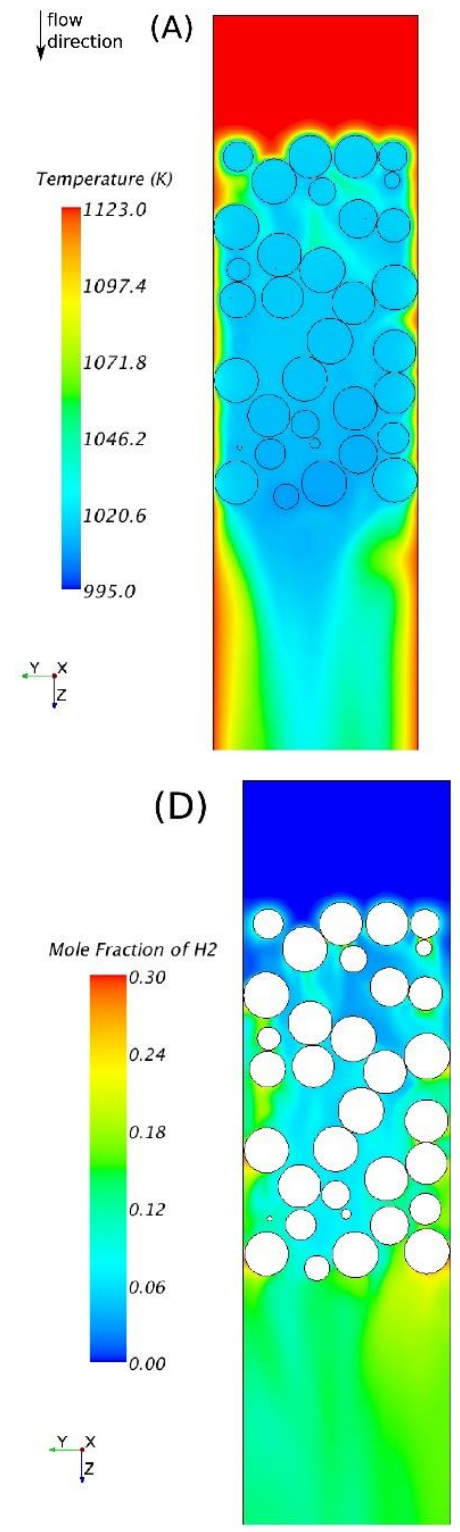

(B)

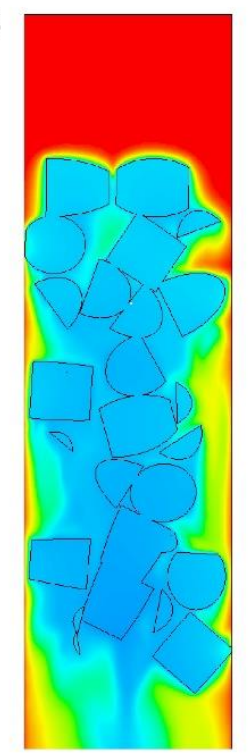

(E)

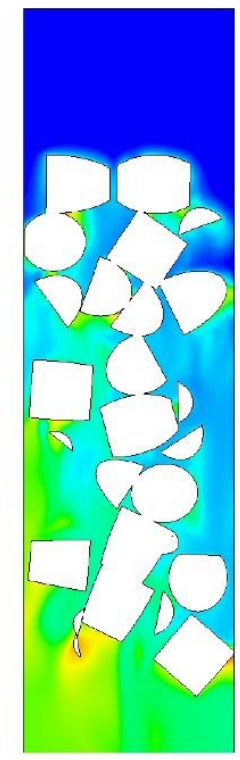

(C)

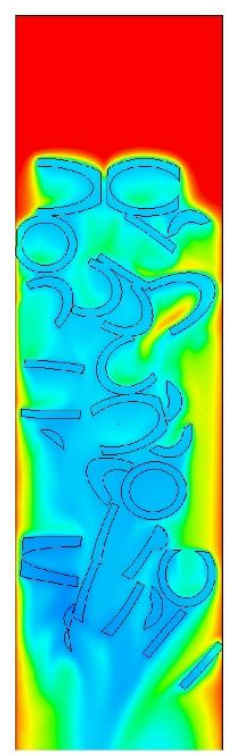

(F)

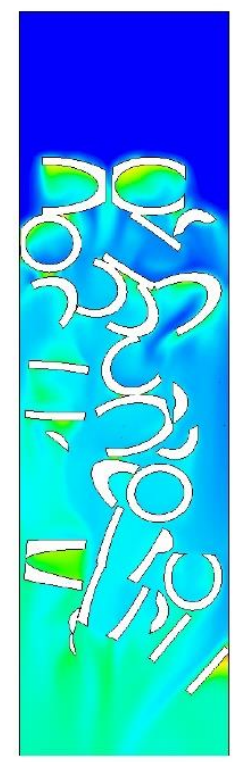

Fig. 10. Modeling of dry reforming of methane in a resolved fixed bed using three different particle shapes. Plane cut through the fixed bed. Temperature distribution: (A) spheres, (B) cylinders, (C) 1-hole cylinders. Mole fraction of hydrogen: (D) spheres, (E) cylinders, (F) 1-hole cylinders (courtesy of G. Wehinger, related paper is: Wehinger 2016) 


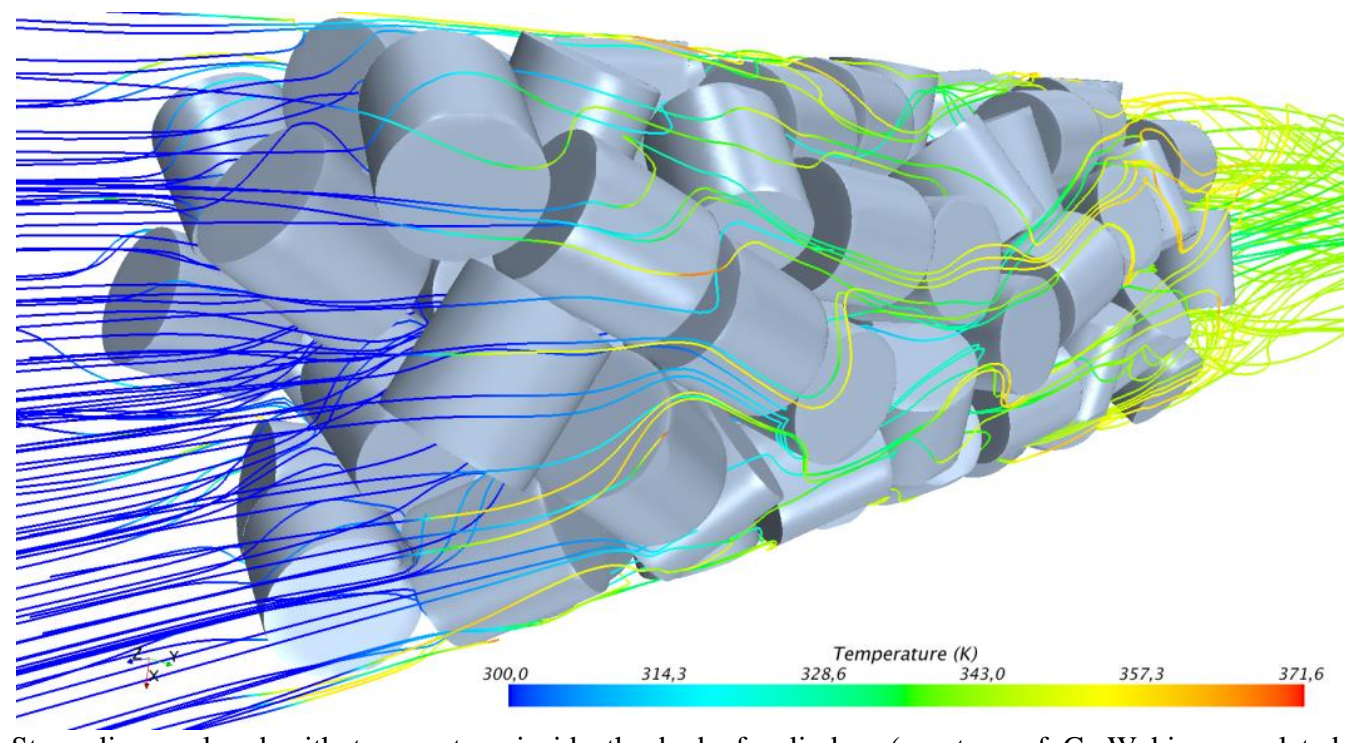

Fig. 11. Streamlines colored with temperature inside the bed of cylinders (courtesy of G. Wehinger, related paper is: Wehinger et al. 2016b)

\subsection{Modelling of mobile catalyst with one fluid phase}

At the present, gas-fluidized beds (FBs) find a widespread application in the petroleum, chemical, metallurgical and energy industries. The large increase in computational resources during recent decades made theoretical approaches for the investigation more applicable, since the governing equations describing FBs can now be solved within accessible time scales. Hence, theoretical modeling of FBs with CFD has become a trend in the field. Three different types of simulation approaches can be distinguished: Direct Numerical Simulations (DNS), Eulerian-Eulerian (EE), and Eulerian-Lagrangian (EL) models. Contrary to DNS, the flow field is not resolved at the particle level in the last approaches mentioned, and consequently closures need to be applied, e.g., for fluid-particle interaction forces.

In DNS, the incompressible Navier-Stokes equations are solved for the gas phase and the flow in the interstices of the particles bed is directly computed. DNS is the most popular tool for developing closure relations required in the averaged models mentioned below. Unfortunately, these simulations are restricted to a few thousand particles. In Eulerian-Eulerian (EE) models, in contrast, the particulate and the gas phase are considered as interpenetrating continua, necessitating additional closure laws. EE models are preferred for the simulation of large-scale devices. Fig. 12 shows an example for the EE-modeling of an industrial scale fluidized bed for olefin polymerization. The height of the calculation domain is about $5 \mathrm{~m}$. 


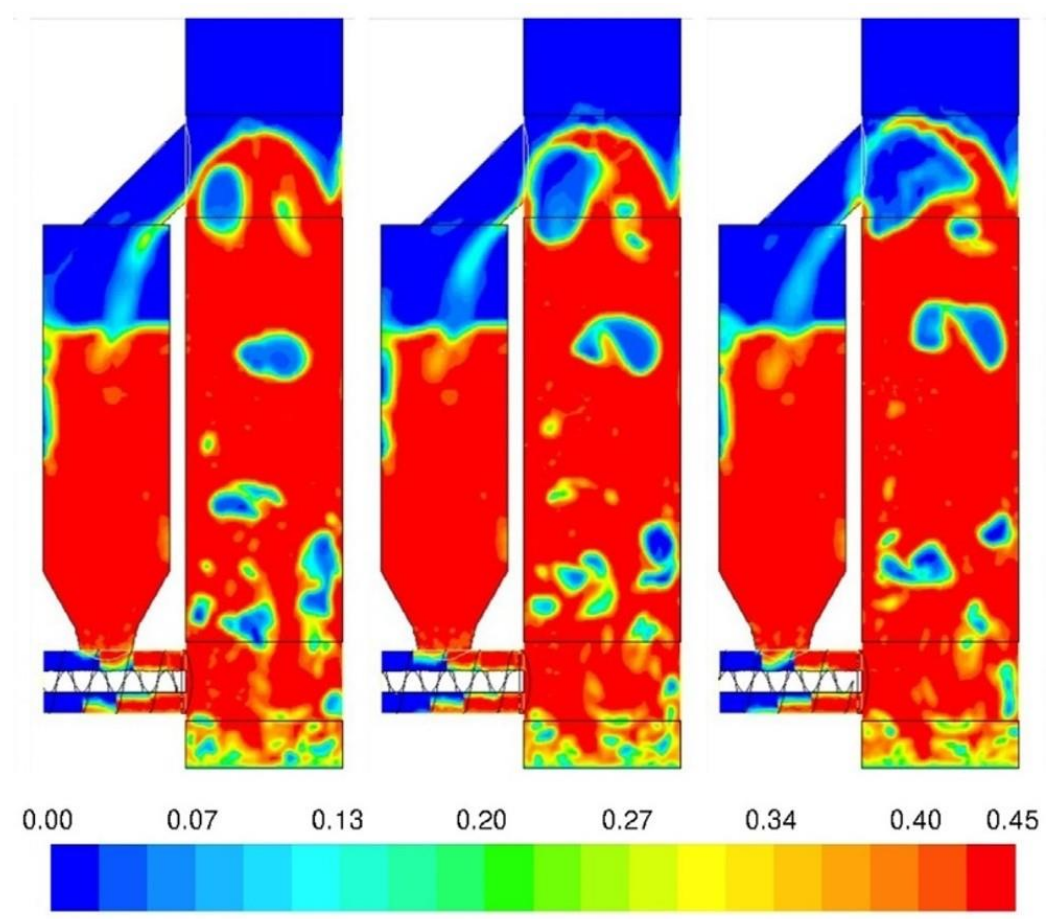

Fig. 12. Snapshots of the solids volume fraction. The time interval between two consecutive pictures is $\Delta t=0.1 \mathrm{~s}$ (Schneiderbauer 2015, reprinted by permission from Elsevier)

The use of an Eulerian-Lagrangian (EL) model (e.g. the Discrete Particle Model, DPM) holds a much greater promise when it comes to the full-physics simulations of heterogeneous reactors. EL models describe the particle phase with discrete entities, e.g., using the discrete element method (DEM) where every particle is tracked individually. EL approaches typically compute the particle-phase interactions directly, and hence do not require complex rheological models and boundary conditions for the granular phase. This opens the door for the application to systems involving non-spherical and irregular particles. While a large number of work focused on inert systems, CFD-DEM calculations accounting for chemical reactions are relatively scarce. The latter are mainly found in the field of biomass and coal conversion, for a recent review see (Zhong et al. 2016). Typically, the employed model assumes a homogeneous concentration and temperature distribution within the particle. This is unrealistic in situations involving fast chemical reactions, small pore diameters, or in case depositions form on the surface of the particles. CFD-DEM calculations accounting for intra-particle transport phenomena are even more rarely done, recently universal open-source tools were developed. Fig. 13 shows an example of the calculation of a fixed bed including surface reactions with an EL approach (Radl et al. 2015). In the left plot, the temperatures of the particles are shown and in the right plot the burst of particles at the upper surface of the bed. Additionally, a part of the CFD grid on the surrounding wall is shown in the right plot. It can be seen, that the particles are not resolved by the grid. Several particles can be in one computational cell. 

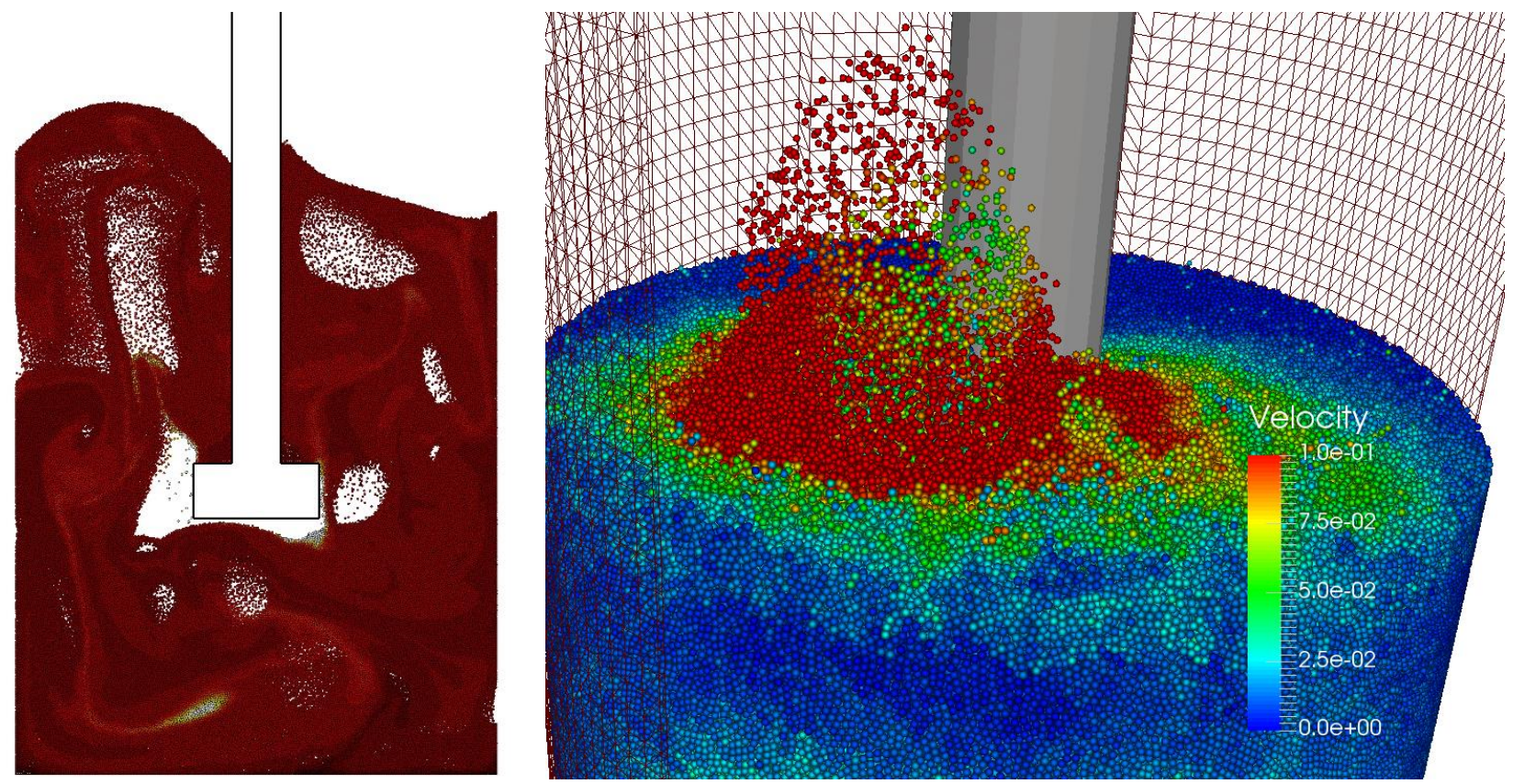

Fig. 13. Modeling of a two-zone fluidized bed reactor for the conversion of butane to butadiene. Left: Snapshot of the particle temperature in a 2D domain (white: $\mathrm{T}=710 \mathrm{~K}$, black: $\mathrm{T}=700 \mathrm{~K}$ ). Diameter/number of particles: $70 \mu \mathrm{m} / 250,000$ (courtesy of Prof. S. Radl, University Graz, Austria). Right: Snapshot of the particle velocity in a 3D domain. Diameter/number of particles: $200 \mu \mathrm{m} / 2,500,000$.

\subsection{Modelling of catalytic reactors with multiple fluid phases}

The catalytic reactors considered so far contain a solid phase (the catalyst and its supports) and one fluid phase (i.e., a gas or a liquid). However, there are also various classes of reactions performed in catalytic reactors containing multiple fluid phases (usually a gas and a liquid, or less often two immiscible liquids) (Önsan and Avci 2016). Examples for heterogeneously catalyzed gas-liquid reactions are hydrogenations, oxidations, hydroformylations, and Fischer-Tropsch synthesis (FTS). The corresponding reactors can be classified in types with immobile catalyst (e.g. fixed beds, trickle beds, solid sponges, monoliths and other structured reactors) and types with significant motion of the catalyst (i.e. slurry reactors such as bubble columns or stirred tanks).

Relevant phenomena in three-phase catalytic reactors arise on multiple scales reflecting multiple physics. The dimensions range from catalyst pores to single particles/bubbles/drops and over particle/bubble clusters to reactor internals toward the entire vessel. Gas-to-liquid processes such as FTS are frequently carried out in slurry reactors (Wang et al. 2007). There, the catalyst particles (diameter 1 to $100 \mu \mathrm{m}$ ) are suspended in the liquid phase while the injected gas rises in the form of bubbles (diameter 1 to $10 \mathrm{~mm}$ ) providing the mixing and catalyst suspension within the vessel (height up to $25 \mathrm{~m}$ ). Coupling of hydrodynamics, heat and mass transfer, and reaction kinetics takes place at molecular and particle levels where conductive and convective transfer and diffusion within the internal pores of the catalyst are accompanied by the adsorption, surface reaction and desorption of reactant and product on the surface.

Full interface-resolving multi-phase CFD simulations of at least some of the relevant phenomena listed above are only possible for small sections of large-scale reactors and for microreactors. In the general case, multi-dimensional CFD approaches relying on a time averaging (Ishii and Hibiki 2006) or volume averaging procedure are indispensable instead (Jakobsen 2008). Mathematically, the 
averaging of the single-phase conservation equations for mass, momentum and energy for all phases yields the Eulerian multi-fluid model, where the phases are represented as interpenetrating continua. For slurry reactors, the liquid and catalyst particles may optionally be represented by one slurry phase with the gas as additional phase.

As the system of averaged Eulerian multi-fluid equations is not closed, constitutive relations must be provided to model turbulence as well as momentum/heat/mass transfer between the phases. Interfacial transfer models (e.g. for the drag force and interfacial heat/mass transfer) are well established for dilute disperse flows (Sommerfeld et al. 2008), however, often relying on idealized situations (e.g. isolated particles moving through stagnant fluid). The development of interfacial transfer and turbulence models for non-dilute and non-disperse flow regimes which are valid in a wide range of operating conditions is actually a larger scientific challenge than the numerical solution of the set of averaged multi-fluid equations themselves (Hjertager 2007).

In its standard form, the Euler-Euler model (two-fluid model) requires input of a (mean) particle/bubble/drop diameter. For narrow size distributions, the Sauter mean diameter may serve well for this purpose. For wider size distributions, which are standard rather than exceptional in technical reactors, various types of population balance approaches are available taking into account e.g. bubble breakup/coalescence (Yeoh et al. 2014; Marchisio and Fox 2013).

A conceptually different approach for disperse flows is the Euler-Lagrange (EL) model, where the continuous phase is still represented in the Eulerian manner while the disperse elements (i.e., particles/drops/bubbles) are described in a Lagrangian way (Sommerfeld 2017; Crowe et al. 2011). There, an equation of motion is solved for a large number of particles or computational parcels yielding particle trajectories. The EL approach is often used for disperse two-phase flows (e.g., bubbly flow or sprays). The interaction between the phases is modelled depending on the disperse volume fraction $\alpha_{P}$, see Fig. 14. For very dilute conditions $\left(\alpha_{P}<5 \cdot 10^{-4}\right)$ one-way coupling is sufficient, where the continuous phase affects the disperse phase but not vice-versa. As $\alpha_{P}$ increases, two-way and fourway coupling are required, where in the latter case also the effects of particle-particle interactions are accounted for. For volume fractions $\alpha_{P}>0.1$, the particle transport is no longer flow/collisions-driven but contact-driven. Such situations are frequently encountered in fluidized beds; they are best described by the discrete element method, in potential combination with CFD (see above).

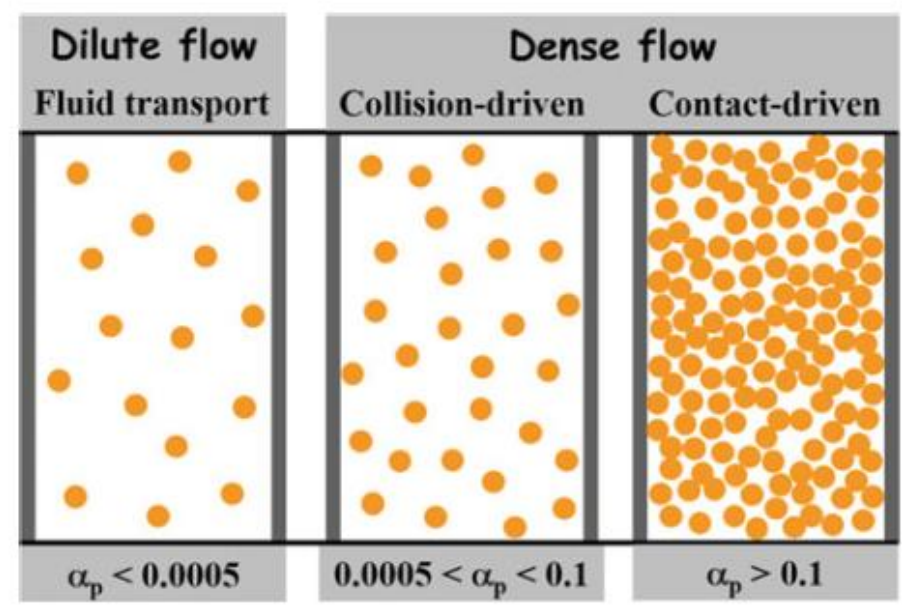

Fig. 14. Regimes of dispersed two-phase flows in terms of transport phenomena; left: dilute flow dominated by fluidtransport of particles; middle: dense flow regime dominated by inter-particle collision; right: dense flow regime that is particle contact dominated (Sommerfeld 2017, reprinted by permission from Springer Nature). 
Three-phase reactors with immobile catalyst are conceptually simpler to model by CFD since the solid domain is invariant in time. The hydrodynamics thus reduces to the simulation of the two-fluid flow coupled with the solid domain by boundary conditions reflecting the conjugate heat transfer and heterogeneous reactions. By a porous body approach (see above), such reactors may be computed on a large scale by the Eulerian multi-fluid model as well. However, reactor details such as a single monolith channel, a section of a trickle bed, a representative part of structured reactors, a portion of a solid foam etc. are well amenable nowadays for interface-resolving simulation of the two-phase flow.

In recent years, microfluidic systems operated in continuous multiphase flows have emerged as useful platforms for synthesizing micro- and nanoparticles with controlled shape and size distribution. They have yielded particles of organic polymers, oxides, semiconductors, and metals as well as hybrid structures combining multiple materials and functionalities (Marre and Jensen 2010). A detailed review on numerical methods for interface-resolving multiphase CFD simulations in microfluidics and micro process engineering (including the widely used volume-of-fluid and level set methods) along with an overview on related applications is given in Wörner (2012). While the number of computational studies combining two-phase hydrodynamics with mass transfer is increasing (especially for segmented gas-liquid flow), numerical studies incorporating chemical kinetics in microreactors in which the catalyst is dispersed on the solid wall are still rare. Here, the study of Woo et al. (2017) on the hydrogenation of nitrobenzene to aniline in gas-liquid Taylor flow within a single channel may serve as an example. The authors first computed the quasi-steady bubble shape and velocity field by a 2D simulation of a single flow unit cell (consisting of one Taylor bubble and liquid slug). Using these (frozen) hydrodynamics, they computed the transient mass transfer of hydrogen from the gas into the liquid phase towards the catalytic wall, where a detailed kinetic mechanism adapted from a density functional theory study is employed, consisting of four bulk species and ten surface species. Fig. 15 illustrates the temporal evolution of educts and product in the bulk phases in combination with wall profiles of all intermediates.
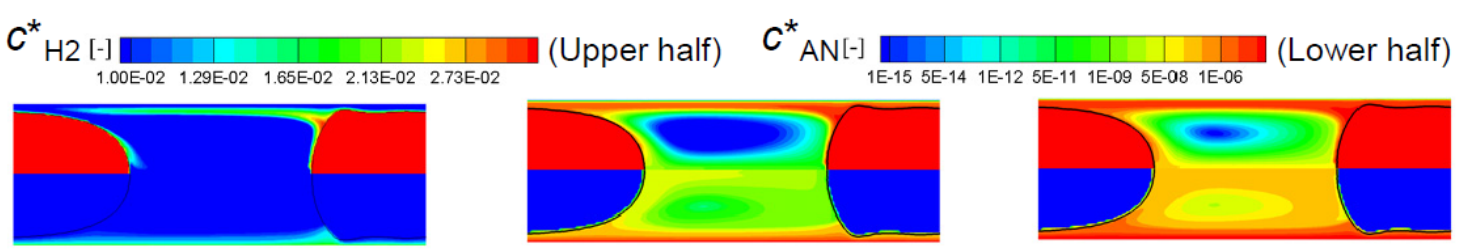

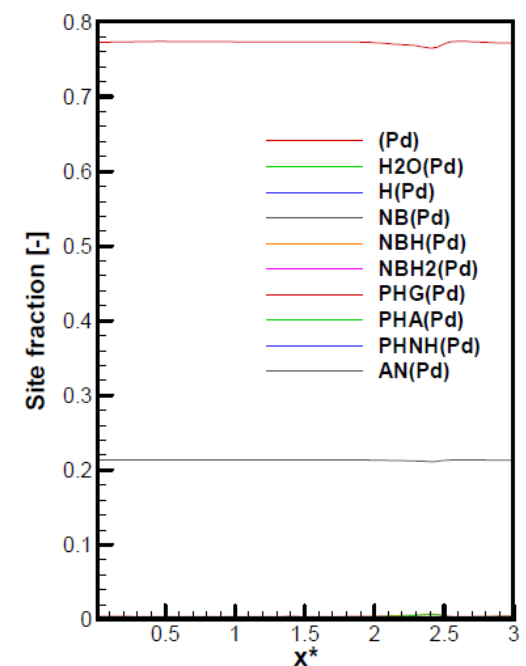

Time $=0.25 \mathrm{~ms}$

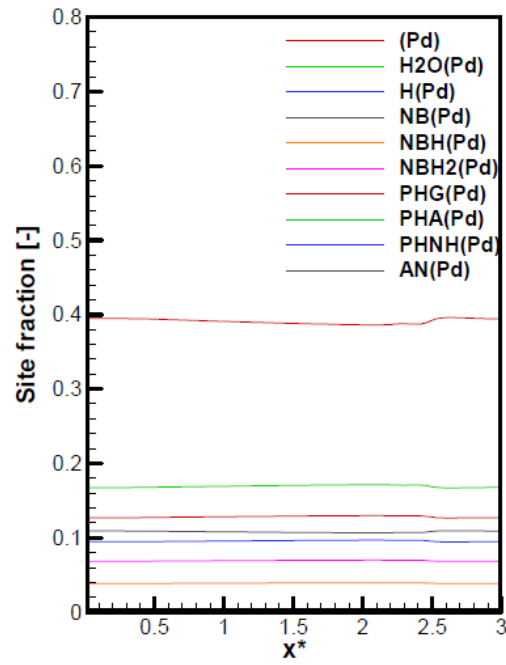

Time $=2.5 \mathrm{~ms}$

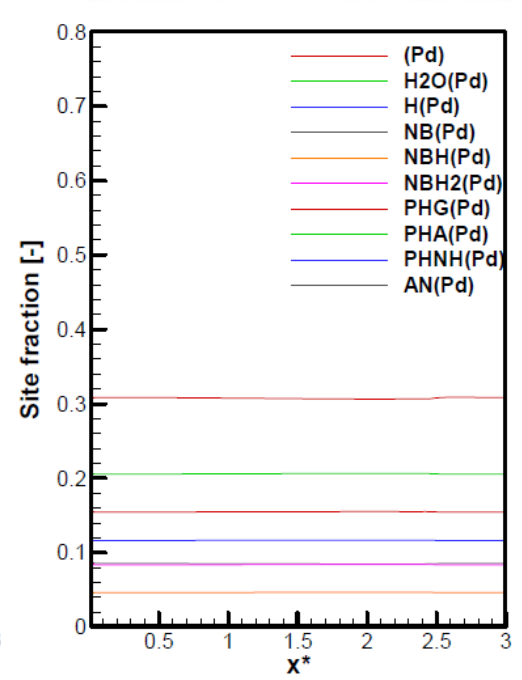

Time $=5 \mathrm{~ms}$ 
Fig. 15. Top row: Normalized instantaneous concentration distributions of hydrogen (upper half of channel) and aniline (lower half of channel) in gas-liquid Taylor flow. Bottom row. Instantaneous wall profiles of intermediate species.

Even though the adequate modeling of physical complexity is challenging for multiphase CFD simulations, computations are a promising tool to achieve a better understanding of three-phase catalytic reactors.

\section{Conclusions and outlook}

Computational Fluid Dynamics or CFD is the analysis of systems involving fluid flow, heat transfer and associated phenomena such as chemical reactions by means of computer-based numerical simulations. The technique is powerful and spans a wide range of industrial and non-industrial applications. The advantages of CFD simulations over experiment-based approaches to reactor system design is the substantial reduction of development times and costs, the ability to study systems where experiments are difficult or impossible, the ability to study systems under hazardous conditions or beyond their normal performance limits and the practically unlimited level of detail of results.

From a reaction engineering perspective, CFD simulations have matured into a powerful tool for understanding mass and heat transport in catalytic reactors. Initially, CFD calculations focused on a better understanding of mixing, mass transfer to enhance reaction rates, diffusion in porous media and heat transfer. Over the last decade, the flow field and heat transport models have also been coupled with models for heterogeneous chemical reactions. So far, most of these models are based on the mean field approximation, in which the local state of the surface is described by its coverage with adsorbed species averaged on a microscopic scale. There is a gap between the length and time scales on the microscopic level and the length and times scales which can be resolved by a computational grid. This gap is bridged with models (e. g. mean field approximation). In the future, the gap has to be shortened due to improved models, the combination of existing and advanced numerical approaches (e. g. Monte-Carlo simulations) and extended computer resources (higher discretization level). The currently increasing research activities on surface reactions at practical conditions will certainly boost the application of CFD codes that combine fluid flow and chemistry. New insights into the complexity of heterogeneous catalysis, however, will also reveal the demand for more sophisticated chemistry models. Their implementation into CFD simulations will then require robust numerical algorithms.

The simulation results will always remain a reflection of the models and physical parameters applied. Various commercial and open source codes are available including a confusing number of methods and models. The careful choice of the sub models (turbulence, diffusion, species, and reactions involved, etc.) and the physical parameters (boundary conditions, properties, etc.) is a precondition for reliable simulation results. Only the use of appropriate models and parameters, which describe all significant processes in the reactor, can lead to reliable results. Numerical algorithms never give the accurate solution of the model equations but only an approximated solution. The solution is always dependent on the calculation grid (calculation domain, discretization level, grid type). Hence, error estimation is needed. Moreover, the practical experience shows that the results from two different codes for the same complex physical problem are not equal.

CFD calculations require careful validation using experimental data or analytical solutions. Not till the model is validated, operational conditions or other parameters should be varied or extended to regions where no data is available. Owning the resources of a CFD program and computer hardware only is 
worthless. Qualified people having long experience in the field of modeling are needed to run the codes and to analyze and interpret the results. The understanding of the physics of fluid flow and the fundamentals of the numerical algorithms is a prerequisite. Having these crucial issues in mind, CFD can really serve as a powerful tool in understanding the behavior in catalytic reactors and in supporting the design and optimization of reactors and processes.

\section{References}

Bird RB, Stewart WE, Lightfoot EN (2001) Transport Phenomena, 2nd ed., John Wiley \& Sons, Inc., New York

Blasi JM, Weddle PJ, Karakaya C, Diercks DR, Kee RJ (2016) Modeling reaction-diffusion processes within catalyst washcoats: II. Macroscale processes informed by microscale simulations. Chem. Eng. Sci. 145:308-316

Choudary C, Mazumder S. (2014) Direct numerical simulation of catalytic combustion in a multi-channel monolith reactor using personal computers with emerging architectures. Computers \& Chemical Engineering, 61, 175-184

Cornejo I, Nikrityuk P, Hayes RE (2018) Multiscale RANS-based modeling of the turbulence decay inside of an automotive catalytic converter, Chemical Engineering Science, 175, 377-386

Crowe CT, Schwarzkopf JD, Sommerfeld M, Tsuji Y. (2011) Multiphase Flows with Droplets and Particles, Second Edition. CRC Press

Dixon AG, Taskin ME, Nijemeisland M, Stitt EH (2011) Systematic mesh development for 3D CFD simulation of fixed beds: Single sphere study. Computers \& Chemical Engineering 35(7):1171-1185

Dudukovic MP (2009) Frontiers in reactor engineering. Science 325:698-701

Dybbs A, Edwards R (1984) A new look at porous media fluid mechanics - darcy to turbulent. In: Bear, J., Corapcioglu, M. (Eds.), Fundamentals of Transport Phenomena in Porous Media. Vol. 82 of NATO ASI Series. Springer Netherlands, pp. $199-256$

Fox RO (2003) Computational methods for turbulent reacting flows. Cambridge University Press

Habisreuther P, Djordjevic N, Zarzalis N (2009) Statistical distribution of residence time and tortuosity of flow through opencell foams. Chemical Engineering Science 64 (23):4943-4954

Hayes RE, Kolaczkowski ST (1997) Introduction to Catalytic Combustion, Gordon and Breach Science Publ., Amsterdam

Hayes RE, Fadic A, Mmbaga J, Najafi A. (2012) CFD modelling of the automotive catalytic converter. Catalysis Today 188:94-105

Hettel M, Diehm C, Bonart H, Deutschmann, O. (2015) Numerical simulation of a structured catalytic methane reformer by DUO: The new computational interface for OpenFOAM ${ }^{\circledR}$ and DETCHEM ${ }^{\mathrm{TM}}$. Catalysis Today 258:230-240.

Hettel M, Daymo E., Deutschmann O (2018) 3D modeling of a CPOX-reformer including detailed chemistry and radiation effects with DUO. Computers and Chemical Engineering 109:166-178

Hjertager BH (2007) Multi-fluid CFD Analysis of Chemical Reactors, In Multiphase Reacting Flows: Modelling and Simulation, Marchisio DL and Fox RO, Editors. Springer, Vienna, pp. 125-179

Ishii M, Hibiki T. (2006) Thermo-fluid dynamics of two-phase flow. Springer, New York, London

Jakobsen HA (2008) Chemical reactor modeling: multiphase reactive flows. Springer, Berlin

Karakaya C, Weddle PJ, Blasi JM, Diercks DR, Kee, RJ (2016) Modeling reaction-diffusion processes within catalyst washcoats: I. Microscale processes based on three-dimensional reconstructions. Chem. Eng. Sci. 145:299-307

Kee RJ, Coltrin ME, Glarborg P (2003) Chemically Reacting Flow, Wiley-Interscience

Kerkhof P, Geboers MAM (2005) American Institute of Chemical Engineering Journal 51, 79.

Korup O, Mavlyankariev S, Geske M, Goldsmith CF, Horn R (2011) Measurement and analysis of spatial reactor profiles in high temperature catalysis research. Chemical Engineering and Processing: Process Intensification 50(10):998-1009.

Kumar A, Mazumder S. (2010) Toward simulation of full-scale monolithic catalytic converters with complex heterogeneous chemistry. Computers \& Chemical Engineering 34:135-145

Kuroki M, Ookawara S, Ogawa K (2009) A high-fidelity CFD model of methane steam reforming in a packed bed reactor. Journal of Chemical Engineering of Japan 42 (Supplement.), s73-s78

Lemos MJS Turbulence in Porous Media, Modeling and Applications (2006) Elsevier

Libby PA and Williams FA (Editors) (1994) Turbulent reacting flows, Academic Press, London

Maestri M, Beretta A, Groppi G, Tronconi E, Forzatti P (2005) Comparison among structured and packed-bed reactors for the catalytic partial oxidation of $\mathrm{CH} 4$ at short contact times. Catalysis Today 105:709-717

Marchisio DL, Fox RO (2013) Computational models for polydisperse particulate and multiphase systems. Cambridge Series in Chemical Engineering. Cambridge University Press, Cambridge

Marre S, Jensen KF (2010) Synthesis of micro and nanostructures in microfluidic systems. Chemical Society Reviews 39:1183-1202

Nien T, Mmbaga JP, Hayes RE, Votsmeier M. (2013) Hierarchical multi-scale model reduction in the simulation of catalytic converters. Chemical Engineering Science 93:362-375

Önsan ZI, Avci AK (2016) Multiphase catalytic reactors theory, design, manufacturing, and applications. John Wiley \& Sons, Inc.

OpenFOAM-The Open Source CFD Toolbox (2017) www.openfoam.org

Oran ES, Boris JP (1987) Numerical Simulation of Reactive Flow. Elsevier

Park HM (2018) A multiscale modeling of fixed bed catalytic reactors, International Journal of Heat and Mass Transfer 116 : $520-531$ 
Patankar SV (1990) Numerical Heat Transfer and Fluid Flow. Series in Computational Methods in Mechanics and Thermal Science. McGraw-Hill

Peters N (2000) Turbulent Combustion. Cambridge University Press, London

Poinsot T, Veynante D (2001) Theoretical and Numerical Combustion. R. T. Edwards, Inc., Philadelphia, PA

Porter S, Saul J, Aleksandrova S, Medina H, Benjamin S (2016) Hybrid flow modelling approach applied to automotive catalysts. Applied Mathematical Modelling 40:8435-8445

Pope SB (2000) Turbulent Flows. Cambridge University Press, London

Radl S, Forgber T, Kloss C, Aigner A (2015) ParScale - A Compilation of Particle Scale Models. Url: https://github.com/CFDEMproject/ParScale-PUBLIC

Schneiderbauer S, Puttinger S, Pirker S, Aguayo P, Kanellopoulos V (2015) CFD modeling and simulation of industrial scale olefin polymerization fluidized bed reactors, Chemical Engineering Journal 264: 99-112

Sharma AK, Birgersson E. (2016) Validity and scalability of an asymptotically reduced single-channel model for full-size catalytic monolith converters. Applied Mathematics and Computation 281:186-198

Sommerfeld M., van Wachem B, Oliemans R (2008) Best practice guidelines for computational fluid dynamics of dispersed multiphase flows. ERCOFTAC, SIAMUF Swedish Industrial Association for Multiphase Flows

Sommerfeld M (2017) Numerical Methods for Dispersed Multiphase Flows, In Particles in Flows, Bodnár T, Galdi GP, Nečasová Š, Editors. Springer International Publishing, Cham, pp. 327-396

Sui R, Prasianakis NI, Mantzaras J, Mallya N, Theile J, Lagrange D, Friess M (2016) An experimental and numerical investigation of the combustion and heat transfer characteristics of hydrogen-fueled catalytic microreactors. Chemical Engineering Science 141:214-230

Tang Y, Lau YM, Deen NG, Peters EAJF, Kuipers JAM (2016) Direct numerical simulations and experiments of a pseudo2D gas-fluidized bed, Chemical Engineering Science 143:166-180

Tischer S, Deutschmann, O (2005) Recent advances in numerical modeling of catalytic monolith reactors. Catalysis Today 105:407-413

Versteeg HK, Malalasekera W (2007) An Introduction to Computational Fluid Dynamics (second ed.): Pearson Education Limited

Wang TF, Wang JF, Jin Y (2007) Slurry reactors for gas-to-liquid processes: A review. Industrial \& Engineering Chemistry Research 46:5824-5847

Warnatz J, Dibble RW, Maas U (1996) Combustion, Physical and Chemical Fundamentals, Modeling and Simulation, Experiments, Pollutant Formation, Springer-Verlag, New York

Wehinger G. (2016) Particle-resolved CFD simulations of catalytic flow reactors, PhD Thesis, Technical University Berlin

Wehinger G, Heitmann H, Kraume M. (2016) An artificial structure modeler for 3D CFD simulations of catalytic foams, Chemical Engineering Journal 284:543-556

Wehinger GD, Kraume M, Berg V, Korup O, Mette K, Schlögl R, Behrens M, Horn R (2016b) Investigating dry reforming of methane with spatial reactor profiles and particle-resolved CFD simulations. AIChE J. 62:4436-4452

Wilcox CC (1998) Turbulence Modeling for CFD. DCW Industries

Wörner M (2012) Numerical modeling of multiphase flows in microfluidics and micro process engineering: a review of methods and applications. Microfluidics and Nanofluidics 12:841-886

Woo M, Wörner M, Maier L, Tischer S, Deutschmann O (2017) A Numerical Study on Gas-Liquid Taylor Flow for Catalytic Hydrogenation of Nitrobenzene with Detailed Kinetic Mechanism, Annual Meeting ProcessNet Section Multiphase flow, March 14-15, 2017, Dresden, Germany, https://doi.org/10.5445/IR/1000068709

Yeoh GH, Cheung CP, Tu J (2014) Multiphase Flow Analysis Using Population Balance Modeling. Butterworth-Heinemann, Oxford

Zhong W, Yu A, Zhou G, Xie J, Zhang H (2016) CFD simulation of dense particulate reaction system: Approaches, recent advances and applications, Chemical Engineering Science 140:16-43 